\title{
The Protonation of Allene and Some Heteroallenes, a Computational Study
}

\author{
Scott Gronert and James R. Keeffe \\ Department of Chemistry, Virginia Commonwealth University, 1001 W. Main St., Richmond, VA \\ 23284 and Department of Chemistry and Biochemistry, San Francisco State University, 1600 \\ Holloway Avenue, San Francisco, California 94132
}

\section{Supporting Information}

1. Table S1. Electronic Energies ${ }^{\mathrm{a}}$, Zero-point Vibrational Energies ${ }^{\mathrm{b}}$, and $\quad$ S2-S7 Transition State (ts) Imaginary Frequencies ${ }^{\mathrm{b}}$ for Structures in This Study

2. Table S2. Cartesian Coordinates for Cumulenes and other Compounds in S8-S34 this Study (MP2/6-311+G** unless otherwise noted).

3. Table S3. Selected Geometric Features of Compounds in This Study ${ }^{\mathrm{a}} \quad$ S35-S38

4. Table S4. G3 Enthalpies

S39-S40 
Table S1. Electronic Energies ${ }^{\text {a }}$ (hartrees), Zero-point Vibrational Energies (kcal/mol), and Transition State (ts) Imaginary Frequencies $^{\mathrm{b}}\left(\mathrm{cm}^{-1}\right)$ for Structures in This Study.

\begin{tabular}{|c|c|c|c|c|c|}
\hline \multirow[b]{2}{*}{ System } & \multicolumn{3}{|c|}{$\mathrm{MP} 2 / 6-311+\mathrm{G}^{* *}$} & \multicolumn{2}{|c|}{ B3LYP/6-31+G** } \\
\hline & Energy & $\mathrm{ZPVE}^{\mathrm{b}}$ & $\mathrm{iv}, \mathrm{cm}^{-1}$ & Energy & ZPVE \\
\hline & \multicolumn{5}{|c|}{$\underline{\text { Allene and Isomers }}$} \\
\hline 1 allene & -116.3081995 & 36.81 & & -116.6716006 & 34.60 \\
\hline 2 propyne & -116.3165964 & 37.33 & & -116.6671914 & 34.84 \\
\hline 3 cyclopropene & -116.2784139 & 37.42 & & -116.6311471 & 35.08 \\
\hline 4 2-propenyl ${ }^{+}$ & -116.5930985 & 42.60 & & -116.9648107 & 39.89 \\
\hline $51+4(t s)$ & -232.9154408 & 78.26 & 1553 & & \\
\hline $\mathbf{5 a} 1+\mathrm{H}_{3} \mathrm{~S}^{+} \rightarrow \mathbf{4}+\mathrm{H}_{2} \mathrm{~S}(\mathrm{ts})$ & -515.4563328 & 52.71 & 1279 & & \\
\hline 6 allyl $^{+}$ & -116.6131729 & 45.40 & & -116.9808831 & 42.92 \\
\hline 7 allyl $^{+}$(rotational ts) & $-116.5542406^{\mathrm{c}}$ & 43.28 & 892 & & \\
\hline $84 \rightarrow 6(t s)^{d}$ & -116.5634150 & 42.14 & 432 & & \\
\hline $91+6(t s)$ & $-232.8959915^{\mathrm{e}}$ & 78.22 & $1126,269^{\mathrm{e}}$ & & \\
\hline 9a $1+\mathrm{H}_{3} \mathrm{~S}^{+} \rightarrow \mathbf{6}+\mathrm{H}_{2} \mathrm{~S}(\mathrm{ts})$ & -515.4417375 & 53.55 & 453 (493 at MP2) & & \\
\hline \multirow[t]{2}{*}{10 cyclopropyl $^{+}$} & $-116.5550835^{\mathrm{f}}$ & 43.23 & 284 (543 at MP2) & -116.9240043 & 41.27 \\
\hline & \multicolumn{5}{|c|}{$\underline{\text { Ketene and Isomers }}$} \\
\hline 11 ketene & -152.2353194 & 21.21 & & -152.6109019 & 19.80 \\
\hline 12 ethynol & -152.1805601 & 21.84 & & -152.5507284 & 19.81 \\
\hline 13 2-oxirene & -152.1057839 & 20.43 & & -152.4882837 & 18.69 \\
\hline 14 acetyl $^{+}$ & -152.5651375 & 29.96 & & -152.9297579 & 27.94 \\
\hline $1511+14(\mathrm{cx})^{\mathrm{g}}$ & -304.8123649 & 51.92 & & & \\
\hline $1611+14(\mathrm{ts})$ & -304.8059336 & 49.20 & 1717 & & \\
\hline $17 \mathrm{CH}_{2}=\mathrm{C}=\mathrm{OH}^{+}$ & -152.4847215 & 29.15 & & -152.868637 & 27.05 \\
\hline 1817 (rotational ts) & -152.4557076 & 29.60 & 1070 & & \\
\hline
\end{tabular}


Table S1. Electronic Energies ${ }^{\text {a }}$ (hartrees), Zero-point Vibrational Energies (kcal/mol), and Transition State (ts) Imaginary Frequencies ${ }^{\mathrm{b}}\left(\mathrm{cm}^{-1}\right)$ for Structures in This Study.

$\mathrm{MP} 2 / 6-311+\mathrm{G}^{* *} \quad \mathrm{~B} 3 \mathrm{LYP} / 6-31+\mathrm{G}^{* *}$ or B3LYP/6-311+G**a

\begin{tabular}{|c|c|c|c|c|c|}
\hline System & Energy & $\mathrm{ZPVE}^{\mathrm{b}}$ & iv, $\mathrm{cm}^{-1}$ & Energy & $\mathrm{ZPVE}^{\mathrm{b}}$ \\
\hline $1911+17(\mathrm{cx})^{\mathrm{g}}$ & -304.7562495 & 51.13 & & & \\
\hline $2011+17(\mathrm{ts})^{\mathrm{g}}$ & -304.7562495 & 48.38 & 1013 & & \\
\hline $21 \mathrm{CH}_{2} \mathrm{CH}=\mathrm{O}^{+}$(O-bridged $)$ & -152.4691871 & 30.23 & & -152.8443196 & 28.05 \\
\hline 2221 (rotational ts) & -152.4226775 & 28.14 & 86 (206 at MP2) & & \\
\hline $2317 \rightarrow 21(\mathrm{ts})$ & -152.3937057 & 22.65 & 2420 & & \\
\hline $2411+21$ (open $\mathrm{C}-\mathrm{C}$ dimer) & )$^{+}-304.8363164$ & 55.95 & & & \\
\hline $25 \begin{array}{l}11+21 \\
\text { (O-bridged C-C dimer) }\end{array}$ & -304.8504505 & 56.29 & $70^{\mathrm{i}}$ & & \\
\hline $26 \begin{array}{l}\mathbf{1 1}+\mathbf{2 1} \\
\text { (H-transfer dimer ts })\end{array}$ & -304.8098030 & 53.04 & $1201^{\mathrm{i}}$ & & \\
\hline
\end{tabular}

$\underline{\text { Ketenimine and Isomers }}$

\begin{tabular}{|c|c|c|c|c|c|}
\hline 27 ketenimine & -132.3577994 & 29.31 & & -132.7269149 & 27.41 \\
\hline 28 acetonitrile & -132.4109416 & 30.31 & & -132.7658127 & 28.41 \\
\hline 29 methyl isonitrile & -132.3673154 & 30.21 & & -132.7301646 & 28.39 \\
\hline 30 ethynamine & -132.3382948 & 30.06 & & -132.69833 & 27.62 \\
\hline 31 2-azirine & -132.2731555 & 29.77 & & -132.6348816 & 27.23 \\
\hline 32 1-azirine & -132.3316227 & 30.27 & & -132.6912416 & 28.21 \\
\hline $33 \mathrm{CH}_{3} \mathrm{C} \equiv \mathrm{NH}^{+}$ & -132.7137202 & 37.64 & & -133.0737295 & 35.09 \\
\hline $3427+33($ ts $)$ & -265.0638890 & 63.24 & 1756 & & \\
\hline $35 \mathrm{CH}_{2}=\mathrm{C}=\mathrm{NH}_{2}^{+}$ & -132.6815273 & 38.25 & & -133.0560042 & 35.80 \\
\hline $3627+35(c x)^{j}$ & -265.0770792 & 68.92 & & & \\
\hline
\end{tabular}


Table S1. Electronic Energies ${ }^{\text {a }}$ (hartrees), Zero-point Vibrational Energies (kcal/mol), and Transition State (ts) Imaginary Frequencies $^{\mathrm{b}}\left(\mathrm{cm}^{-1}\right)$ for Structures in This Study.

\begin{tabular}{|c|c|c|c|c|c|c|}
\hline \multirow[b]{2}{*}{$\underline{\text { System }}$} & \multicolumn{3}{|c|}{$\mathrm{MP} 2 / 6-311+\mathrm{G}^{* *}$} & \multicolumn{3}{|c|}{ B3LYP/6-31+G** } \\
\hline & Energy & $\mathrm{ZPVE}^{\mathrm{b}}$ & iv, $\mathrm{cm}^{-1}$ & Energy & $\mathrm{ZPVE}^{\mathrm{b}}$ & iv \\
\hline $3727+35(t s)^{j}$ & -265.0765207 & 66.18 & 1420 & & & \\
\hline $38 \mathrm{CH}_{2} \mathrm{CH}=\mathrm{NH}^{+}$(open) & -132.6030357 & 37.58 & & -132.9789355 & 35.19 & \\
\hline $39 \mathrm{CH}_{2} \mathrm{CHNH}^{+}$(N-bridged) & -132.6446242 & 38.60 & & -133.0102414 & 36.08 & \\
\hline $40 \mathrm{CH}_{3} \mathrm{~N} \equiv \mathrm{CH}^{+}$ & -132.6978560 & 38.07 & & -133.0562249 & 35.59 & \\
\hline $41 \mathrm{HC} \equiv \mathrm{C}-\mathrm{NH}_{3}{ }^{+}$ & -132.6537516 & 40.16 & & -133.0111924 & 37.12 & \\
\hline \multicolumn{7}{|c|}{ Diazomethane, Carbodiimide, and Isomers } \\
\hline 42 diazomethane & -148.3759000 & 21.24 & & -148.7503928 & 19.97 & \\
\hline 43 1-diazirine & -148.3630527 & 22.73 & & -148.7295819 & 21.00 & \\
\hline 44 2-diazirine & -148.3274050 & 22.42 & & -148.6949739 & 20.40 & \\
\hline 45 carbodiimide & -148.4220690 & 21.87 & & -148.7958231 & 20.43 & \\
\hline $46 \mathrm{CH}_{3} \mathrm{~N} \equiv \mathrm{N}^{+}$ & -148.7310891 & 30.35 & & -149.0891265 & 28.42 & \\
\hline $4742+46(\mathrm{cx})^{\mathrm{k}}$ & -297.1242457 & 52.37 & & & & \\
\hline $4842+46(\mathrm{ts})^{\mathrm{k}}$ & -297.1181851 & 49.53 & 1701 & & & \\
\hline $49 \mathrm{CH}_{2} \mathrm{NNH}^{+}$(bent at N1) & -148.6597703 & $30 / 28$ & & -149.0436929 & 27.95 & \\
\hline $50 \mathrm{CH}_{2} \mathrm{NNH}^{+}$(linear at N1) & -148.6229867 & 28.42 & 1230 & & & \\
\hline $5142+49(\mathrm{cx})^{\mathrm{k}}$ & -297.0628830 & 52.88 & & & & \\
\hline $5242+49(\mathrm{ts})^{\mathrm{k}}$ & -297.0616940 & 50.14 & 1512 & & & \\
\hline $53 \mathrm{CH}_{2}=\mathrm{NHN}^{+}$ & -148.5062594 & 29.76 & & -148.904622 & 26.95 & \\
\hline 53a $\mathrm{CH}_{2} \mathrm{NHN}^{+}$(N-bridged) & -148.6320548 & 31.06 & & -149.0031008 & 28.73 & \\
\hline $54 \mathrm{NH}_{2}=\mathrm{C}=\mathrm{NH}^{+}$ & -148.7440579 & 29.89 & & -149.115694 & 28.13 & \\
\hline $5545+54(\mathrm{cx})^{1}$ & -297.2004084 & 52.99 & & & & \\
\hline $5645+54(\mathrm{ts})^{1}$ & -297.1995111 & 50.34 & 1366 & & & \\
\hline $57 \mathrm{HNCHNH}^{+}$(open) & -148.6114521 & 29.35 & & $-149.0225949^{a}$ & 27.29 & \\
\hline
\end{tabular}


Table S1. Electronic Energies ${ }^{\text {a }}$ (hartrees), Zero-point Vibrational Energies (kcal/mol), and Transition State (ts) Imaginary Frequencies ${ }^{\mathrm{b}}\left(\mathrm{cm}^{-1}\right)$ for Structures in This Study.

$\mathrm{MP} 2 / 6-311+\mathrm{G}^{* *} \quad \mathrm{~B} 3 \mathrm{LYP} / 6-31+\mathrm{G}^{* *}$ or B3LYP/6-311+G**a

\begin{tabular}{|c|c|c|c|c|c|}
\hline System & Energy & $\mathrm{ZPVE}^{\mathrm{b}}$ & $\mathrm{iv}, \mathrm{cm}^{-1}$ & Energy & $\mathrm{ZPVE}^{\mathrm{t}}$ \\
\hline $58 \mathrm{CHNHNH}^{+}$(C-bridged) & -148.6114516 & 29.27 & & -148.9892249 & 27.38 \\
\hline $5945+57(\text { open cx })^{\mathrm{m}}$ & -297.1323621 & 57.07 & & & \\
\hline $6059(\text { H-transfer ts })^{\mathrm{m}}$ & -297.0754890 & 53.57 & 1271 (653 at MP2) & & \\
\hline 61 cyanamide & -148.4326087 & 23.05 & & -148.7970365 & 21.32 \\
\hline 62 cyanamide $(\mathrm{ts})^{\mathrm{n}}$ & -148.4300439 & 22.32 & 435 (562 at MP2) & & \\
\hline $63 \mathrm{HN} \equiv \mathrm{C}-\mathrm{NH}_{2}{ }^{+}$(equals 54) & -148.7440579 & 29.90 & & -149.115694 & 28.13 \\
\hline $64 \mathrm{~N} \equiv \mathrm{C}-\mathrm{NH}_{3}{ }^{+}$ & -148.7113622 & 32.67 & & -149.0712433 & 30.23 \\
\hline
\end{tabular}

Carbon Dioxide

\begin{tabular}{|c|c|c|c|c|c|}
\hline 65 carbon dioxide & -188.2065580 & 8.05 & & -188.5903926 & 7.26 \\
\hline 66 carbon dioxide, cyclic & -187.9648023 & 4.72 & & -188.3627004 & 4.23 \\
\hline $67 \mathrm{OCOH}^{+}$ & -188.4197035 & 14.67 & & -188.8006515 & 13.41 \\
\hline $6865+67(c x)$ & -376.6580908 & 23.36 & & & \\
\hline $6965+67($ ts $)$ & -376.6580905 & 20.65 & 719 & & \\
\hline $70 \mathrm{OCOH}^{+} \rightarrow{ }^{+} \mathrm{HOCO}(\mathrm{ts})$ & -188.2818121 & 10.29 & 2275 & & \\
\hline $71 \mathrm{OCHO}^{+}$ & -188.2522810 & 13.39 & & -188.6373656 & 12.58 \\
\hline $7265+71(\mathrm{O}-\mathrm{H}-\mathrm{C} \mathrm{cx})^{\mathrm{o}}$ & -376.4492216 & 23.38 & & & \\
\hline $\begin{array}{c}7365+71(\mathrm{O}-\text {-bridged } \\
\text { dimer, } \mathrm{C}-\mathrm{H}-\mathrm{C} \text { ts })^{\mathrm{O}}\end{array}$ & -376.4122015 & 22.08 & $1193^{\circ}$ & & \\
\hline
\end{tabular}


Table S1. Electronic Energies ${ }^{\text {a }}$ (hartrees), Zero-point Vibrational Energies (kcal/mol), and Transition State (ts) Imaginary Frequencies ${ }^{\mathrm{b}}\left(\mathrm{cm}^{-1}\right)$ for Structures in This Study.

\begin{tabular}{|c|c|c|c|c|c|}
\hline \multirow[b]{2}{*}{ System } & \multicolumn{3}{|c|}{$\mathrm{MP} 2 / 6-311+\mathrm{G}^{* *}$} & \multicolumn{2}{|c|}{ B3LYP/6-31+G** or B3LYl } \\
\hline & Energy & $\mathrm{ZPVE}^{\mathrm{b}}$ & iv, $\mathrm{cm}^{-1}$ & Energy & ZPVE \\
\hline & & & cyanic A & & \\
\hline 74 isocyanic acid, $\mathrm{HN}=\mathrm{C}=\mathrm{O}$ & -168.3117852 & 14.24 & & -168.6912769 & 13.30 \\
\hline $75 \mathrm{H}_{2} \mathrm{~N}-\mathrm{C} \equiv \mathrm{O}^{+}$ & -168.5999879 & 22.86 & & -168.9747658 & 21.26 \\
\hline $76 \mathrm{HN} \equiv \mathrm{C}-\mathrm{OH}^{+}$ & -168.5692829 & 22.04 & & -168.947383 & 20.11 \\
\hline $77 \mathrm{HNCHO}^{+}$ & -168.439922 & 21.82 & & -168.8233879 & 20.02 \\
\hline
\end{tabular}

$\underline{\mathrm{CH}}_{2}-\underline{\mathrm{B}-\mathrm{CH}_{2}} \underline{2}^{-}$

\begin{tabular}{|c|c|c|c|c|c|}
\hline $78 \mathrm{CH}_{2} \mathrm{BCH}_{2}^{-\mathrm{q}}$ & -103.1006201 & 33.25 & & -103.4541947 & 31.54 \\
\hline $79 \mathrm{CH}_{2} \mathrm{BCH}_{2}^{-}$(planar, ts $)^{\mathrm{q}}$ & -103.0576594 & 30.73 & 1033 & & \\
\hline $80 \mathrm{CH}_{3} \mathrm{BCH}_{2}^{\mathrm{r}}$ & -103.6833377 & 41.33 & & -104.0383828 & 39.13 \\
\hline $81 \mathrm{CH}_{2} \mathrm{BHCH}_{2}(\mathrm{ts})^{\mathrm{s}}$ & -103.6335016 & 41.09 & 192 & & \\
\hline 82 borirane, $\left(\mathrm{CH}_{2} \mathrm{BHCH}_{2}\right)^{\mathrm{s}}$ & -103.6680583 & 41.87 & & -104.0198818 & 39.47 \\
\hline $8378+80(t s)^{t}$ & -206.7854155 & 72.69 & 1815 & & \\
\hline
\end{tabular}

${ }^{\mathrm{a}} \mathrm{MP} 2 / 6-311+\mathrm{G}^{* *}$ or B3LYP/6-31+G**. ${ }^{\mathrm{b}}$ The listed ab initio ZPVE values are for the HF/6-311+G** geometries. The listed DFT ZPVEs are at the level indicated for the electronic energies. The use of HF ZPVE values in place of MP2 values has been found to cause insignificant differences in over $100 \Delta \mathrm{H}$ calculations: This work and see, Gronert, S.; Keeffe, J. R. J. Org. Chem. 2006, 77, 5959. ${ }^{\mathrm{c}} \mathrm{MP} 2 / 6-$

$311+\mathrm{G}^{* *} / / \mathrm{HF} / 6-311+\mathrm{G}^{* *}$. A "perpendicular" allyl cation could not be found at MP2/6-311+G**. 'The transition state for the rearrangement of 2-propenyl ${ }^{+}$to allyl ${ }^{+}$. ${ }^{\mathrm{e}}$ The transition state for proton transfer from $\mathrm{C} 2$ of allyl ${ }^{+}$to $\mathrm{C} 2$ of allene. A fully optimized structure was not obtained. Structure 9 has the partial C-H distances constrained to $1.420 \AA$. It is a second-order saddle point. ${ }^{\mathrm{f}} \mathrm{Cyclopropyl}$ cation, 10, is a transition state for disrotatory ring opening to allyl ${ }^{+}, \mathbf{6}$. ' Structure $\mathbf{1 5}$ is the $\mathrm{C} 3-\mathrm{H}-\mathrm{C} 3$ ' hydrogen-bridged ion-molecule complex, and $\mathbf{1 6}$ is the corresponding proton-transfer ts. 19 is the $\mathrm{O}-\mathrm{H}-\mathrm{O}$ hydrogen-bridged ion-molecule complex, and $\mathbf{2 0}$ is the corresponding proton-transfer ts. ${ }^{\mathrm{h}}$ Structure 22 is flat. It is the ts for rotation about the C-C bond of structure $\mathbf{2 1}$. ${ }^{\mathrm{i}}$ In structure $\mathbf{2 5}$ the oxygen in moiety $\mathbf{2 1}$ is proximal to the $\mathrm{C}=\mathrm{O}$ carbon of the ketene moiety. In structure $\mathbf{2 6}$ hydride transfer between carbonyl carbons takes place. MP $2 / 6-311+\mathrm{G}^{* *}$ frequencies show this structure to be a stable H-bridged complex with no imaginary frequency. ${ }^{j}$ Structure 36 is the N-H-N hydrogen-bridged ion-molecule 
complex. Structure 37 is the corresponding proton-transfer ts. ${ }^{\mathrm{k}}$ Structure $\mathbf{4 7}$ is the C-H-C hydrogen-bridged ion-molecule complex, and $\mathbf{4 8}$ is the corresponding proton-transfer ts. Structure $\mathbf{5 1}$ is the N1-H-N1' hydrogen-bridged ion-molecule complex, and $\mathbf{5 2}$ is the corresponding proton-transfer ts. 'Structure $\mathbf{5 5}$ is the N-H-N hydrogen-bridged ion-molecule complex, and $\mathbf{5 6}$ is the corresponding proton-transfer ts.

${ }^{\mathrm{m}}$ Structure 59 is an open dimer with an N-N bond. Structure $\mathbf{6 0}$ is a cyclic ts with the N-N bond and with hydride transfer between carbons taking place. ${ }^{\mathrm{n}}$ Structure $\mathbf{6 2}$ is the ts for flattening about the $-\mathrm{NH}_{2}$ nitrogen. ${ }^{\circ}$ Structure $\mathbf{7 2}$ is an open dimer with an O-O bond. Structure $\mathbf{7 3}$ is dimeric with an $\mathrm{O}-\mathrm{O}$ bond and with hydride transfer occurring between the carbons. At $\mathrm{MP} 2 / 6-311+\mathrm{G}^{* *}$ there is no imaginary frequency; the structure is a dimer with symmetrical hydrogen-bridging between carbons. ${ }^{\mathrm{p}}$ Structure 77 has a short N-O distance. These structures are transition states for disrotatory ring opening of the ring-closed isomer. There is no imaginary frequency at the MP2 level. ${ }^{\mathrm{q}} \mathrm{Structure} \mathbf{7 8}$ has allenic D2 symmetry; $\mathbf{7 9}$ is the planar ts for rotation about a C-B bond. ${ }^{\mathrm{r}}$ Structure $\mathbf{8 0}$, the carbon-protonated conjugate acid of $\mathbf{7 8}$, is linear about the C-B-C axis. ${ }^{\text {s}}$ Transition state 81, protonated on boron, is a singlet with allyl ${ }^{+}$symmetry. Structure $\mathbf{8 2}$ has the shape of cyclopropyl

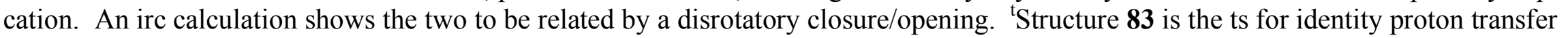
between $\mathbf{7 8}$ and $\mathbf{8 0}$. 
Table S2. Cartesian Coordinates for Cumulenes and other Compounds in this_Study (MP2/6-311+G** or DFT= B3LYP/6-31+G).

$\begin{array}{lrrr}\text { 1. allene (MP2) } & & \\ \mathrm{C} & .000000 & .000000 & -1.314016 \\ \mathrm{C} & .000000 & .000000 & .000000 \\ \mathrm{C} & .000000 & .000000 & 1.314016 \\ \mathrm{H} & -.658983 & .658969 & 1.871005 \\ \mathrm{H} & .658983 & -.658969 & 1.871005 \\ \mathrm{H} & .658983 & .658969 & -1.871005 \\ \mathrm{H} & -.658983 & -.658969 & -1.871005\end{array}$

1, allene (DFT)

C 0. 0. -1.3089402198

C 0. 0. 0 .

C 0. 0. 1.3089402198

H $-0.656992537 \quad 0.6574302279 \quad 1.8735054275$

H $0.656992537-0.65743022791 .8735054276$

H $0.656992537 \quad 0.657430228-1.8735054275$

H $-0.6569925369-0.657430228-1.8735054276$

2. propyne (MP2)

$\begin{array}{lccc}\mathrm{H} & .511621 & .886154 & 1.632995 \\ \mathrm{C} & .000000 & .000000 & 1.248667 \\ \mathrm{H} & .511621 & -.886154 & 1.632995 \\ \mathrm{C} & .000000 & .000000 & -.215158 \\ \mathrm{H} & -1.023242 & .000000 & 1.632995 \\ \mathrm{C} & .000000 & .000000 & -1.433639 \\ \mathrm{H} & .000000 & .000000 & -2.498205\end{array}$

2. propyne (DFT)

H $0.5114481626 \quad 0.88585420311 .6336339543$

C 0. 0. 1.2420613543

H $0.5114481626-0.8858542031 \quad 1.6336339543$

C $0.0 . \quad-0.2188856664$

$\mathrm{H}-1.0228963252 \quad 0.1 .6336339543$

C 0. 0. -1.4289731107

H $0.0 .-2.4949095479$

3. cyclopropene (MP2)

H $-1.5861674178 \quad 0 .-1.0403599895$

H $0 .-0.91624394041 .4553399457$

H $1.5861674178 \quad 0 .-1.0403599895$

C -0.6527764547 0. -0.5016813528

$\begin{array}{llll}C & 0.6527764547 & 0 . & -0.5016813528\end{array}$

C 0. 0.0 .8650360535 
H 0.0 .91624394041 .4553399457

3. cyclopropene (DFT)

H $-1.5815013584 \quad 0$. -1.0447540586

H $0 .-0.91485335621 .4620261995$

H $1.5815013584 \quad 0 .-1.0447540586$

C $-0.64827711110 .-0.5033038617$

$\begin{array}{llll}C & 0.6482771111 & 0 . & -0.5033038617\end{array}$

C 0.0 .0 .863696702

H 0.0 .91485335621 .4620261995

$\begin{array}{lrrr}\text { 4. 2-propenyl } \mathrm{l}^{+}(\mathrm{MP} 2) & & \\ \mathrm{H} & -0.277328 & 1.208661 & -1.581677 \\ \mathrm{C} & -0.068637 & 1.199643 & -0.508602 \\ \mathrm{H} & 0.998741 & 1.502856 & -0.380671 \\ \mathrm{C} & -0.028543 & -0.101958 & 0.035103 \\ \mathrm{H} & -0.671265 & 1.892538 & 0.084582 \\ \mathrm{C} & 0.059836 & -1.271965 & 0.536208 \\ \mathrm{H} & -0.835712 & -1.901506 & 0.582858 \\ \mathrm{H} & 1.009629 & -1.656869 & 0.918650\end{array}$
4, 2-propenyl ${ }^{+}$(DFT)
H $-0.27868761961 .2088650489-1.5796172777$
C $-0.05862909161 .1966026191-0.5049829614$
H $0.99643036121 .5439989776-0.3981107376$
$\begin{array}{llll}\text { C } & -0.0185746374 & -0.1038408872 & 0.0381845817\end{array}$
$\begin{array}{llll}\mathrm{H} & -0.6716781983 & 1.8910530082 & 0.0826618312\end{array}$
C $0.0556791235-1.2744537703 \quad 0.5361581699$
H $-0.8349616682-1.9124888108 \quad 0.5875792968$
H $0.9971427305-1.67833618540 .9245780971$

5. allene + 2-propenyl $1^{+}$, proton transfer ts (MP2)

$\begin{array}{lrrr}\mathrm{H} & -1.962674 & 0.892660 & -0.098466 \\ \mathrm{C} & -1.632071 & 0.143277 & 0.621292 \\ \mathrm{H} & -0.228613 & 0.422357 & 0.622388 \\ \mathrm{C} & -1.435543 & -1.120683 & 0.201798 \\ \mathrm{H} & -1.873899 & 0.314784 & 1.669980 \\ \mathrm{C} & -1.105861 & -2.312543 & -0.198073 \\ \mathrm{H} & -0.061613 & -2.591505 & -0.331401 \\ \mathrm{H} & -1.876709 & -3.053717 & -0.400461 \\ \mathrm{C} & 1.025132 & 0.978027 & 1.031026 \\ \mathrm{H} & 1.397095 & 0.152305 & 1.638273 \\ \mathrm{H} & 0.689499 & 1.873194 & 1.554159 \\ \mathrm{C} & 1.449972 & 1.094026 & -0.241075 \\ \mathrm{C} & 1.732616 & 1.154641 & -1.508181 \\ \mathrm{H} & 1.013588 & 0.832763 & -2.260141\end{array}$


5a. allene $+\mathrm{H}_{3} \mathrm{~S}^{+}$, proton tansfer to $\mathrm{C} 1$ ts (MP2)

H $3.5414849485-0.08255806690 .0143789455$

$\begin{array}{llll}\text { C } 2.5026995204 & 0.2468014641 & 0.0270459778\end{array}$

H $2.2819865837 \quad 1.31284773510 .0846405171$

C $1.5518498093 \quad-0.6256800081 \quad-0.0260275287$

C $0.4512781232-1.4202493777-0.0756258236$

H $0.1978870121-1.98228543160 .8240068165$

H $0.207714659-1.8799202556-1.0341717419$

H $-0.3453130868 \quad-0.4186362666-0.0247072845$

S - $1.745841401 \quad 0.8272413120 .0360517458$

H $-2.4980576388 \quad 0.2513010370 .9804626339$

H $-2.4872047794 \quad 0.3581577864 \quad-0.9737935719$

6. allyl $^{+}(\mathrm{MP} 2)$

$\begin{array}{lrrr}\mathrm{H} & -1.172129 & 0.000000 & -1.303592 \\ \mathrm{C} & -1.184117 & 0.000000 & -0.214528 \\ \mathrm{C} & 0.000000 & 0.000000 & 0.505422 \\ \mathrm{H} & -2.153741 & 0.000000 & 0.279359 \\ \mathrm{C} & 1.184117 & 0.000000 & -0.214528 \\ \mathrm{H} & 0.000000 & 0.000000 & 1.590266 \\ \mathrm{H} & 2.153741 & 0.000000 & 0.279359 \\ \mathrm{H} & 1.172129 & 0.000000 & -1.303592\end{array}$

6. allyl $^{+}(\mathrm{DFT})$

H -1.2062013968 0. -1.3040764136

C -1.1920971588 0. -0.2148965565

C 0. 0. 0.4899771324

H -2.1540970917 0. 0.2948100521

C 1.1920971588 0. -0.2148965565

H 0.0 .1 .5765147034

H 2.1540970917 0. 0.2948100521

H 1.2062013968 0. -1.3040764136

7. allyl $^{+}$, rotational ts, $\mathrm{HF} / 6-311+\mathrm{G}^{* *}$

$\mathrm{H} \quad-1.385023 \quad-1.214260 \quad 0.008703$

$\begin{array}{llll}\text { C } & -0.309738 & -1.205772 & 0.002224\end{array}$

$\begin{array}{llll}\text { C } & 0.397727 & -0.095472 & -0.002364\end{array}$

$\mathrm{H} \quad \begin{array}{llll}\mathrm{H} & 0.192045 & -2.154925 & -0.000520\end{array}$

$\begin{array}{llll}\mathrm{C} & -0.067838 & 1.270316 & 0.000029\end{array}$

$\begin{array}{lllll}\mathrm{H} & 1.489971 & -0.088512 & -0.008946\end{array}$

$\mathrm{H} \quad \begin{array}{llll}-0.214507 & 1.821354 & -0.922305\end{array}$

$\mathrm{H} \quad-0.203389 \quad 1.821910 \quad 0.923732$

8. 2-propenyl ${ }^{+} \rightarrow$ allyl $^{+}$, hydride shift ts (MP2) 


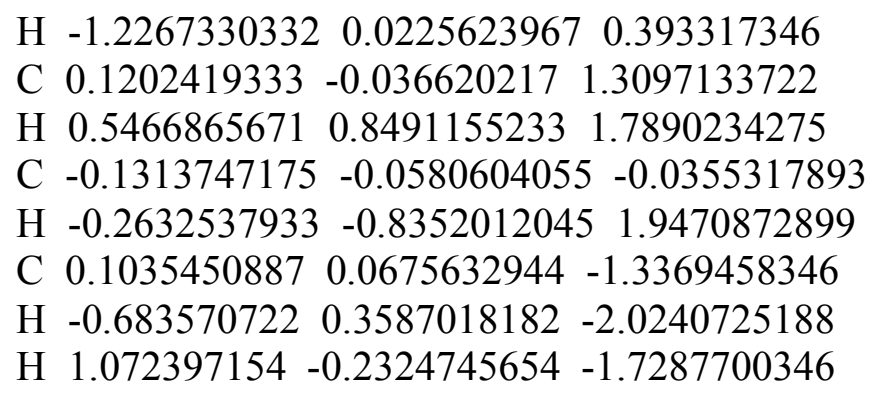

9. allene + allyl ${ }^{+}$, proton transfer ts, $\mathrm{d}(\mathrm{C} . . . \mathrm{H})$ constrained to $1.420 \AA$ (MP2)

$\begin{array}{lrrr}\mathrm{H} & -1.718872 & -0.095661 & -2.444743 \\ \mathrm{C} & -1.136689 & 0.407336 & -1.673549 \\ \mathrm{C} & 0.057219 & -0.073255 & -1.310410 \\ \mathrm{H} & -1.604031 & 1.229080 & -1.133950 \\ \mathrm{C} & 1.307675 & -0.518907 & -1.416251 \\ \mathrm{H} & -0.203046 & -0.279962 & 0.070146 \\ \mathrm{H} & 1.716521 & -1.256879 & -0.730806 \\ \mathrm{H} & 1.978290 & -0.074469 & -2.148977 \\ \mathrm{C} & -0.078696 & 0.091605 & 1.435018 \\ \mathrm{C} & 0.444221 & 1.284418 & 1.598886 \\ \mathrm{C} & -0.579664 & -1.150187 & 1.412664 \\ \mathrm{H} & 0.843956 & 1.851096 & 0.763599 \\ \mathrm{H} & 0.465243 & 1.715989 & 2.596462 \\ \mathrm{H} & 0.075181 & -2.013279 & 1.532622 \\ \mathrm{H} & -1.637639 & -1.321974 & 1.217500\end{array}$

9a. allene $+\mathrm{H}_{3} \mathrm{~S}^{+}$, proton tansfer to $\mathrm{C} 2$ ts (MP2)

H $0.91857816311 .5592076517-1.4446981839$

C $1.60386345810 .8697017583 \quad-0.9561599809$

C 1.21448693420 .15982986420 .1252871647

H $2.54874003540 .6551938558-1.4537620973$

H $-0.0388553123-0.02918489640 .0015588207$

C $1.6093360204-0.4192210604 \quad 1.2801219023$

H $1.1089003614-1.3084690631 .6568234237$

H $2.33073952390 .0709358874 \quad 1.9326752879$

S $-1.8334777628-0.1879738526-0.2595003617$

H $-2.2127911163-0.0763034147 \quad 1.0194729185$

H $-1.8857859269-1.5256617508-0.2555588987$

10. cyclopropyl ${ }^{+}$(MP2)

$\begin{array}{lrrr}\mathrm{H} & 0.607817 & 1.318325 & 0.915558 \\ \mathrm{C} & 0.374005 & 0.768142 & -0.001035 \\ \mathrm{C} & 0.374005 & -0.768142 & -0.001035 \\ \mathrm{C} & -0.830880 & 0.000000 & 0.002347 \\ \mathrm{H} & 0.601143 & 1.319875 & -0.918376 \\ \mathrm{H} & 0.607817 & -1.318325 & 0.915558\end{array}$




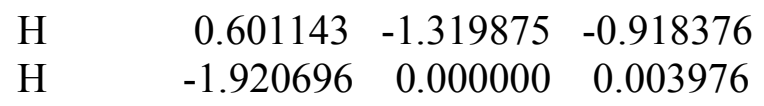

10. cyclopropyl ${ }^{+}$(DFT)

H $0.6130470088 \quad 1.3297788340 .9097237452$

$\begin{array}{lllll}\text { C } & 0.3673155459 & 0.769083108 & -0.0009469863\end{array}$

C $0.3673155459-0.769083108-0.0009469863$

C -0.83241183020 .0 .0024378419$

H $0.6048669081 \quad 1.3325632365 \quad-0.9120856741$

Н $0.6130470088-1.3297788340 .9097237452$

H $0.6048669081-1.3325632365-0.9120856741$

H -1.92369309120 .0 .0027969881$

11. ketene (MP2)

H $\quad 0.943447 \quad 0.000000 \quad-1.743613$

$\begin{array}{lllll}\text { C } & 0.000000 & 0.000000 & -1.218150\end{array}$

$\begin{array}{llll}\text { C } & 0.000000 & 0.000000 & 0.103576\end{array}$

$\mathrm{H} \quad-0.943447 \quad 0.000000 \quad-1.743613$

O $\quad 0.000000 \quad 0.000000 \quad 1.271834$

11. ketene (DFT)

H $0.9412811216 \quad 0 . \quad-1.7476324413$

C 0. 0. -1.2129470224

C 0. 0. 0.1033972218

H $-0.94128112160 .-1.7476324413$

O 0.0 .1 .2748486832

12. ethynol (MP2)

$\begin{array}{llll}\mathrm{H} & 0.020258 & 0.000000 & -2.394833\end{array}$

$\begin{array}{lllll}\text { C } & 0.001426 & 0.000000 & -1.332588\end{array}$

$\begin{array}{llll}\text { C } & -0.008111 & 0.000000 & -0.119140\end{array}$

$\begin{array}{llll}\mathrm{O} & -0.096990 & 0.000000 & 1.193579\end{array}$

$\mathrm{H} \quad 0.795768 \quad 0.000000 \quad 1.556562$

12. ethynol (DFT)

H $0.012717768 \quad 0 .-2.4002727771$

C $0.0003106376 \quad 0 .-1.3360340684$

C $-0.00339638920 .-0.128143815$

O -0.0901040747 0. 1.1842006617

H $0.7928230584 \quad 0.1 .5838299989$

13. 2-oxirene (MP2)

H 1.63386094530 .55477531130$.

C 0.63082365240 .17744555010 .

C -0.64476763320 .10581071030$.

O $0.0613023976-1.21733676920$. 
H - 1.68121930910 .37930519760$.

13. 2-oxirene (DFT)

H 1.57746811650 .37216947540 .3348966435

$\begin{array}{llll}\text { C } & 0.7576520579 & 0.316763091 & -0.3819924063\end{array}$

C -0.51486417320 .00829096630 .026013051$

$\begin{array}{lllll}\text { O } & -0.3566326478 & -1.2441409434 & 0.1035953398\end{array}$

H -1.46362335340 .54691741070 .117487372$

14. acetyl $\mathrm{l}^{+}$(MP2)

$\mathrm{H}$

$\begin{array}{lll}521475 & .903222 & 1.561507\end{array}$

$\begin{array}{llll}\mathrm{C} & .000000 & .000000 & 1.225053\end{array}$

$\begin{array}{llll}\mathrm{H} & .521475 & -.903222 & 1.561507\end{array}$

$\begin{array}{llll}\mathrm{C} & .000000 & .000000 & -.215699\end{array}$

$\mathrm{H} \quad \begin{array}{llll}\mathrm{C} & -1.042950 & .000000 & 1.561507\end{array}$

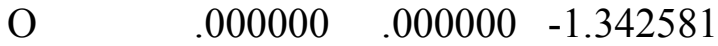

14. acetyl ${ }^{+}$(DFT)

H 0.52161448140 .90346278381 .5637459139

C 0. 0. 1.2154367955

H $0.5216144814 \quad-0.9034627838 \quad 1.5637459139$

C 0. $0 .-0.2167549651$

H -1.04322896280 .1 .5637459139$

O $0.0 .-1.3407359571$

15. ketene + acetyl $^{+}$, ion-molecule complex (MP2)

$\begin{array}{lrrr}\mathrm{H} & 1.561368 & -0.937119 & -1.255668 \\ \mathrm{C} & 1.106773 & -1.272182 & -0.328336 \\ \mathrm{H} & 1.715044 & -1.274230 & 0.571038 \\ \mathrm{C} & 0.276774 & -2.320563 & -0.451972 \\ \mathrm{H} & -0.277208 & 0.289682 & 0.076087 \\ \mathrm{O} & -0.513190 & -3.161241 & -0.540635 \\ \mathrm{C} & -0.997106 & 1.127203 & 0.291092 \\ \mathrm{C} & -0.225213 & 2.316678 & 0.447092 \\ \mathrm{H} & -1.539933 & 0.945422 & 1.225171 \\ \mathrm{H} & -1.687445 & 1.273547 & -0.546897 \\ \mathrm{O} & 0.420791 & 3.235726 & 0.563512\end{array}$

16. ketene + acetyl $^{+}$, proton transfer ts (MP2)

$\mathrm{H} \quad 0.393335 \quad-1.257742 \quad-1.513137$

C $\quad 0.573986 \quad-1.206325-0.437150$

$\mathrm{H} \quad \begin{array}{llll}\mathrm{C} & 1.631388 & -1.142851 & -0.170864\end{array}$

$\begin{array}{llll}\mathrm{C} & -0.037372 & -2.252164 & 0.216304\end{array}$

$\mathrm{H} \quad-0.005708 \quad-0.000494 \quad-0.006221$

$\begin{array}{llll}\mathrm{O} & -0.616543 & -3.033470 & 0.817885\end{array}$ 


$\begin{array}{lrrr}\mathrm{C} & -0.587412 & 1.205196 & 0.422432 \\ \mathrm{C} & 0.038719 & 2.252313 & -0.214784 \\ \mathrm{H} & -0.424499 & 1.249885 & 1.501552 \\ \mathrm{H} & -1.640466 & 1.147304 & 0.138263 \\ \mathrm{O} & 0.631347 & 3.034692 & -0.801686\end{array}$

$\begin{array}{lrrr}\text { 17. } \mathrm{H}_{2} \mathrm{C}=\mathrm{C}=\mathrm{OH}^{+} & (\mathrm{MP} 2) \\ \mathrm{H} & 1.014819 & 0.011488 & -1.789802 \\ \mathrm{C} & 0.046660 & -0.012497 & -1.288359 \\ \mathrm{C} & 0.000415 & 0.009664 & -0.006286 \\ \mathrm{H} & -0.884770 & -0.029146 & -1.855607 \\ \mathrm{O} & -0.041866 & -0.091680 & 1.214740 \\ \mathrm{H} & -0.077577 & 0.768092 & 1.695361\end{array}$

17. $\mathrm{H}_{2} \mathrm{C}=\mathrm{C}=\mathrm{OH}^{+}$(DFT)

H $1.01046606860 .0039491314-1.805385424$

C $0.0464406082 \quad-0.0138919653-1.2906348609$

C $0.00029086740 .0224380501 \quad-0.011050347$

H $-0.878964112-0.0365592922-1.8718436738$

O $-0.0419054758 \quad-0.080826703 \quad 1.2152733838$

H -0.07864695640 .76081177891 .7336879219$

18. $\mathrm{H}_{2} \mathrm{C}=\mathrm{C}=\mathrm{OH}^{+}$, rotational ts (MP2)

$\mathrm{H} \quad 0.888221 \quad 0.000000 \quad-1.883245$

C $\quad-0.029839 \quad 0.000000 \quad-1.292861$

$\begin{array}{llll}\text { C } & 0.026599 & 0.000000 & -0.016101\end{array}$

$\mathrm{H} \quad-1.009785 \quad 0.000000 \quad-1.778592$

$\begin{array}{llll}\mathrm{O} & -0.061580 & 0.000000 & 1.203098\end{array}$

$\begin{array}{llll}\mathrm{H} & 0.633646 & 0.000000 & 1.890827\end{array}$

19. ketene $+\mathrm{H}_{2} \mathrm{C}=\mathrm{C}=\mathrm{OH}^{+}$. ion $=$molecule complex (MP2)

H $3.7600632615-0.483371956-0.9371647757$

C $3.2677437977 \quad-0.25932702220 .0042602093$

$\begin{array}{llll}\text { C } 2.0649587057 & 0.2199553409 & -0.0084470014\end{array}$

H $3.7622237191 \quad-0.42809235750 .9560224294$

O $0.967182031 \quad 0.7091106261 \quad-0.0217809091$

H $0.00042676260 .0000854506-0.0001109199$

O $\quad-0.9672521828 \quad-0.7092994858 \quad 0.021507472$

$\begin{array}{llll}\text { C } & -2.0649112219 & -0.219804453 & 0.0085671363\end{array}$

C $-3.267779852 \quad 0.2593829863 \quad-0.0040484915$

H $-3.7620459607 \quad 0.4283195039-0.955887596$

H $\quad-3.76017514490 .48332912510 .9373372425$

20. ketene $+\mathrm{H}_{2} \mathrm{C}=\mathrm{C}=\mathrm{OH}^{+}$, proton transfer ts (MP2)

H $-2.096705351-1.183509297-3.0742757279$

C $-2.32883671-1.1210057524-2.0155000202$ 
C $-1.3611411917-1.0579700494-1.1576357552$

H $-3.3548086593-1.1120199341-1.6604010311$

$\begin{array}{lllll}\mathrm{O} & -0.4630353806 & -1.0463830349 & -0.3591527445\end{array}$

H $0.00005904820 .000274471 \quad-0.0000408284$

$\begin{array}{lllll}\text { O } & 0.463123024 & 1.0467851692 & 0.3590258817\end{array}$

C $1.36113955491 .0582084991 \quad 1.157622767$

C $2.3287559645 \quad 1.1204267667 \quad 2.0156269974$

H 3.35477523481 .11122148161 .6606707087

H 2.09647287441 .18285942073 .0743778469

$\begin{array}{lrcc}\text { 21. } & \mathrm{CH}_{2} \mathrm{CH}=\mathrm{O}^{+} & (\mathrm{O}-\text { bridged }) & (\mathrm{MP} 2) \\ \mathrm{O} & -0.066185 & -0.117536 & -0.840923 \\ \mathrm{C} & 0.501139 & 0.529092 & 0.533479 \\ \mathrm{H} & 1.527839 & 0.223661 & 0.709584 \\ \mathrm{C} & -0.492728 & -0.471065 & 0.271110 \\ \mathrm{H} & 0.198783 & 1.567696 & 0.624762 \\ \mathrm{H} & -1.247606 & -1.199237 & 0.565505\end{array}$

21. $\mathrm{CH}_{2} \mathrm{CH}=\mathrm{O}^{+}$(O-bridged) (DFT)

O $-0.0673788901 \quad-0.119803809-0.8413166116$

$\begin{array}{lllll}\text { C } & 0.5007653311 & 0.5284974994 & 0.5308306337\end{array}$

H $1.53041542790 .2264412968 \quad 0.7116235863$

$\begin{array}{llll}\text { C } & -0.493439102 & -0.4713099877 & 0.2677575918\end{array}$

H $0.200943414 \quad 1.57003090620 .6277885377$

H $-1.250064181-1.20124490550 .5668332621$

22. $\mathrm{CH}_{2} \mathrm{CHO}^{+}$, rotational ts (MP2)

$\begin{array}{llll}\mathrm{H} & 1.246601 & 0.000000 & -1.222763\end{array}$

$\begin{array}{llll}\text { C } & 0.154028 & 0.000000 & -1.169775\end{array}$

$\begin{array}{llll}\mathrm{C} & -0.465582 & 0.000000 & 0.182187\end{array}$

$\mathrm{H} \quad-0.429822 \quad 0.000000 \quad-2.094635$

$\begin{array}{llll}\mathrm{O} & 0.326133 & 0.000000 & 1.119877\end{array}$

$\mathrm{H} \quad-1.556525 \quad 0.000000 \quad 0.283908$

23. $\mathrm{CH}_{2}=\mathrm{C}=\mathrm{OH}^{+} \rightarrow \mathrm{CH}_{2} \mathrm{CH}=\mathrm{O}^{+}$(O-bridged), proton shift ts (MP2)

H $1.5960504576-0.8013201108 \quad 0.9556212426$

C $1.1675457091-0.49685446090$.

C -0.03482771730 .07547126980$.

H $1.5960504576-0.8013201108-0.9556212426$

H $-0.9308549095-0.76035831710$.

O -1.13219424460 .61141221060$.

24. ketene $+\mathrm{CH}_{2} \mathrm{CH}=\mathrm{O}^{+}$(O-bridged), open $\mathrm{C}-\mathrm{C}$ dimmer (MP2)

C $1.3171127522 \quad 1.0210843323 \quad 0.6113710334$

C $0.36477037770 .375022681-0.2504220801$ 

H $-0.2102614164 \quad 1.1717567131 \quad-0.7422945475$
C $-0.5484339025-0.5966984917 \quad 0.5336973496$
H $0.941641289-0.1424431844-1.0294432634$
H $0.0290581746-1.39189384641 .0168031399$
C $-1.5062216123-1.212919305-0.4901787787$
H $-1.1255737-0.0747811731 \quad 1.3042382422$
O $2.06066209331 .5208326331 \quad 1.2998387534$
H $-2.2314129261-1.9372590979-0.0838080255$
O $-1.4565142322 \quad-0.913871972-1.6613763397$

25. ketene $+\mathrm{CH}_{2} \mathrm{CH}=\mathrm{O}^{+}$(O-bridged), oxygen-bridged $\mathrm{C}-\mathrm{C}$ dimmer (MP2)

O $-1.0520728266-0.5677947836-0.5754455603$

C $1.1279178413 \quad 0.3492673509 \quad 0.3956862441$

H $1.91939247880 .3752895496 \quad-0.3635028444$

C $0.7746710251-1.0778471867 \quad 0.8128058287$

H 1.42550735160 .99690190661 .2258027051

H $1.5717348811 \quad-1.79218821890 .5870968623$

C $-0.4751298405-1.44487262410 .0670712878$

H $0.5631338548-1.16124695481 .886205069$

C $-0.0401414241 .0026951918-0.2509862601$

H $-0.8903562979-2.45772509230 .0959239935$

O $\quad-0.5620919083 \quad 1.9507338359 \quad-0.6219279883$

26. ketene $+\mathrm{CH}_{2} \mathrm{CH}=\mathrm{O}^{+}$(O-bridged), hydrogen-bridged $\mathrm{C}-\mathrm{C}$ dimmer (MP2)

C $-0.7545588396-0.0705312553-1.1246682529$

C $0.75455883960 .0705312553-1.1246682529$

H $1.2749357594-0.6825332993-1.7233577303$

C $1.2195349298-0.0942480705 \quad 0.3171147938$

H $1.09120008351 .068156457-1.4316211148$

C $-1.21953492980 .0942480705 \quad 0.3171147938$

H - $1.0912000835-1.068156457-1.4316211148$

H $-1.27493575940 .6825332993-1.7233577303$

O $-2.1846048153 \quad 0.2516016603 \quad 0.9415252191$

H 0.0 .0 .9361956934

O $2.1846048153 \quad-0.2516016603 \quad 0.9415252191$

27. ketenimine (MP2)

$\begin{array}{lrcc}\mathrm{C} & -0.015419 & -0.058182 & -1.258429 \\ \mathrm{C} & 0.001016 & 0.016307 & 0.058313 \\ \mathrm{H} & -0.959425 & -0.054890 & -1.787307 \\ \mathrm{H} & 0.915707 & -0.093793 & -1.808525 \\ \mathrm{~N} & 0.013261 & -0.062816 & 1.289959 \\ \mathrm{H} & 0.037308 & 0.839651 & 1.766821\end{array}$

27. ketenimine (DFT)

C $-0.0153548436-0.0560208621-1.2561838235$ 
C 0.00088317790 .01324709330 .057027656

H $-0.9566664185-0.0579415751-1.7936087084$

H $0.9127147581-0.0966016859-1.8146967483$

N $0.0132826256 \quad-0.0539097852 \quad 1.2845663398$

H $0.0375887005 \quad 0.8375038151 \quad 1.7837272844$

28. acetonitrile (MP2)

$\begin{array}{llll}\mathrm{H} & 0.513199 & 0.888886 & 1.558460\end{array}$

$\begin{array}{llll}\mathrm{C} & 0.000000 & 0.000000 & 1.187146\end{array}$

$\begin{array}{llll}\mathrm{H} & 0.513199 & -0.888886 & 1.558460\end{array}$

$\begin{array}{llll}\mathrm{C} & 0.000000 & 0.000000 & -0.275598\end{array}$

$\begin{array}{llll}\mathrm{H} & -1.026397 & 0.000000 & 1.558460\end{array}$

$\begin{array}{llll}\mathrm{N} & 0.000000 & 0.000000 & -1.449238\end{array}$

28. acetonitrile (DFT)

H 0.51356649540 .88952318441 .5581560643

C 0.00000004550 .1 .1827096356

H $0.5135664954-0.8895231844 \quad 1.5581560643$

C $0.0000000455 \quad 0 .-0.2793388307$

$\mathrm{H}-1.0271328545 \quad 0.1 .5581560643$

N $0.00000004550 .-1.4402146307$

29. methyl isonitrile (MP2)

H 0.51479838790 .89165696351 .4770457364

C 0. 0. 1.1161794456

H $0.5147983879-0.89165696351 .4770457364$

N 0. 0. -0.3096212178

H -1.02959677580 .1 .4770457364$

C 0. 0. -1.4934775597

29. methyl isonitrile (DFT)

H $0.5144295814 \quad 0.89101817191 .480123833$

C 0. 0. 1.1098404611

H $0.5144295814-0.89101817191 .480123833$

$\mathrm{N}$ 0. $0 .-0.313139215$

H -1.02885916280 .1 .480123833$

C 0. 0. -1.4880035375

30. ethynamine (MP2)

H $0.04813795480 .0818715999-2.437817435$

C $0.03334744460 .0605988234-1.374971084$

$\begin{array}{llll}\text { C } & 0.0041764261 & 0.0050102999 & -0.1591615201\end{array}$

N $0.01476804480 .0253450766 \quad 1.2036366531$

H -0.91082986430 .08983185931 .607908301$

H $0.5341723712-0.7427737357 \quad 1.6092481868$ 
30. ethynamine (DFT)

H $0.05663789840 .0962936194-2.4410118243$

C $0.02917464460 .051029186-1.3777317436$

$\begin{array}{llll}\text { C } & 0.0002083282 & 0.0009943927 & -0.1669354675\end{array}$

N $-0.0031997957-0.0053506392 \quad 1.1868361335$

$\begin{array}{llll}\mathrm{H} & -0.9093561077 & 0.1090492031 & 1.6240568394\end{array}$

H $0.5503074094-0.7321318386 \quad 1.6236291642$

31. 2-azirene (MP2)

$\mathrm{N}-0.8841545666 \quad 0.0 .185786279$

$\begin{array}{llll}\text { C } & 0.4777681024 & 0.6436550751 & -0.0480811974\end{array}$

H $0.9096055556-1.6267497179-0.0014335044$

H $0.90960555561 .6267497179-0.0014335044$

H $-1.3633463741 \quad 0 .-0.720662576$

$\begin{array}{llll}\text { C } & 0.4777681024 & -0.6436550751 & -0.0480811974\end{array}$

31. 2-azirene (DFT)

$\mathrm{N}-0.8830633993 \quad 0.0 .1809073601$

$\begin{array}{lllll}\text { C } & 0.4802774403 & 0.6393778648 & -0.039708497\end{array}$

H $0.9116339518-1.624572643-0.0060974387$

H $0.91163395181 .624572643 \quad-0.0060974387$

H $-1.3735130071 \quad 0 .-0.7232011992$

$\begin{array}{llll}\text { C } & 0.4802774403 & -0.6393778648 & -0.039708497\end{array}$

32. 1-azirene (MP2)

$\begin{array}{lllll}\text { C } & 0.323923859 & 0 . & 0.7855688391\end{array}$

H $0.89873314650 .-1.603545894$

H 0.4541772020 .92481651121 .3381027789

$\begin{array}{llll}\text { C } & 0.3586172779 & 0 . & -0.6662890447\end{array}$

N $-0.8431906246 \quad 0 .-0.2554769187$

H $0.454177202-0.92481651121 .3381027789$

32. 1-azirene (DFT)

C $0.3162963226 \quad 0.0 .7842204027$

H $0.8958265190 .-1.6087703279$

H $0.45559945020 .9225604766 \quad 1.3441773768$

$\begin{array}{llll}\mathrm{C} & 0.3548642141 & 0 . & -0.6688876233\end{array}$

N -0.8317478933 0. -0.2584546655

H $0.4555994502-0.9225604766 \quad 1.3441773768$

33. $\mathrm{CH}_{3} \mathrm{C} \equiv \mathrm{NH}^{+}(\mathrm{MP} 2)$

$\begin{array}{llll}\mathrm{H} & 0.518396 & 0.897888 & 1.630057\end{array}$

$\begin{array}{llll}\text { C } & 0.000000 & 0.000000 & 1.281950\end{array}$

$\begin{array}{llll}\mathrm{H} & 0.518396 & -0.897888 & 1.630057\end{array}$

$\begin{array}{llll}\mathrm{C} & 0.000000 & 0.000000 & -0.167029\end{array}$

$\begin{array}{llll}\mathrm{H} & -1.036792 & 0.000000 & 1.630057\end{array}$ 
$\begin{array}{llll}\mathrm{N} & 0.000000 & 0.000000 & -1.320772 \\ \mathrm{H} & 0.000000 & 0.000000 & -2.334291\end{array}$

33. $\mathrm{CH}_{3} \mathrm{C} \equiv \mathrm{NH}^{+}$(DFT)

H $0.51804218190 .8972753795 \quad 1.6313333522$

C 0. 0. 1.2723302011

H $0.5180421819-0.89727537951 .6313333522$

C 0. 0. -0.1692145141

$\mathrm{H}-1.0360843638$ 0. 1.6313333522

N 0. $0 .-1.317380676$

H 0. $0 .-2.3286018242$

34. ketenimine $+\mathrm{CH}_{3} \mathrm{C} \equiv \mathrm{NH}^{+}$, proton transfer ts (MP2)

$\begin{array}{llll}\mathrm{H} & -0.064812 & 1.982867 & 0.048711\end{array}$

$\begin{array}{llll}\mathrm{C} & -0.695188 & 1.172341 & -0.318671\end{array}$

$\mathrm{H} \quad-0.000172 \quad-0.000100 \quad 0.000016$

$\begin{array}{lllll}\text { C } & -0.830903 & 1.184457 & -1.691387\end{array}$

$\begin{array}{llll}\mathrm{H} & -1.651619 & 1.082809 & 0.197588\end{array}$

$\begin{array}{llll}\mathrm{N} & -0.815138 & 0.964160 & -2.853147\end{array}$

$\mathrm{H} \quad-1.089731 \quad 1.297318 \quad-3.766440$

$\begin{array}{llll}\text { C } & 0.694797 & -1.172570 & 0.318708\end{array}$

$\mathrm{H} \quad \begin{array}{llll}\mathrm{C} & 1.651081 & -1.083262 & -0.197861\end{array}$

$\begin{array}{llll}\mathrm{H} & 0.064198 & -1.983077 & -0.048329\end{array}$

$\begin{array}{llll}\text { C } & 0.830928 & -1.184442 & 1.691385\end{array}$

$\begin{array}{llll}\mathrm{N} & 0.815546 & -0.963924 & 2.853107\end{array}$

$\mathrm{H} \quad 1.090398 \quad-1.296921 \quad 3.766382$

35. $\mathrm{CH}_{2}=\mathrm{C}=\mathrm{NH}_{2}^{+}(\mathrm{MP} 2)$

$\mathrm{H} \quad-0.945012 \quad 0.000501 \quad-1.874730$

$\begin{array}{llll}\mathrm{C} & 0.000000 & 0.000000 & -1.334939\end{array}$

$\begin{array}{lllll}\mathrm{C} & 0.000000 & 0.000000 & -0.042959\end{array}$

$\mathrm{H} \quad 0.945012 \quad-0.000501-1.874730$

$\begin{array}{llll}\mathrm{N} & 0.000000 & 0.000000 & 1.217960\end{array}$

$\begin{array}{llll}\mathrm{H} & -0.000579 & -0.875382 & 1.745567\end{array}$

$\begin{array}{llll}\mathrm{H} & 0.000580 & 0.875382 & 1.745567\end{array}$

35. $\mathrm{CH}_{2}=\mathrm{C}=\mathrm{NH}_{2}^{+}$(DFT)

H $-0.94089429370 .0000115694-1.8834922879$

C -0.0000000283 0. -1.3324699066

C $0.0000000411 \quad 0 .-0.0440562729$

H 0.9408941779 - $0.0000115694-1.8834923892$

$\mathrm{N} 0.0000001091 \quad 0.1 .2182205854$

H $-0.0011103677 \quad-0.8724038977 \quad 1.7535130844$

H $0.00111064340 .8724038977 \quad 1.7535130843$

36. ketenimine $+\mathrm{CH}_{2}=\mathrm{C}=\mathrm{NH}_{2}{ }^{+}$, ion-molecule complex (MP2) 


$\begin{array}{lrrr}\mathrm{H} & -1.463295 & -0.913220 & -3.158480 \\ \mathrm{C} & -0.418710 & -1.083766 & -2.915030 \\ \mathrm{C} & -0.066081 & -1.267224 & -1.679619 \\ \mathrm{H} & 0.330502 & -1.117378 & -3.700617 \\ \mathrm{~N} & 0.282161 & -1.392137 & -0.480818 \\ \mathrm{H} & 0.302007 & -2.327838 & -0.070017 \\ \mathrm{H} & 0.577233 & -0.501514 & 0.145067 \\ \mathrm{~N} & 0.931545 & 0.698432 & 0.976056 \\ \mathrm{H} & 1.878306 & 1.083741 & 1.028356 \\ \mathrm{C} & 0.086308 & 1.281830 & 1.686300 \\ \mathrm{C} & -0.870703 & 1.821569 & 2.392417 \\ \mathrm{H} & -1.052378 & 1.480371 & 3.405470 \\ \mathrm{H} & -1.453202 & 2.637323 & 1.979144\end{array}$

37. ketenimine $+\mathrm{CH}_{2}=\mathrm{C}=\mathrm{NH}_{2}{ }^{+}$, proton transfer ts (MP2)

$\begin{array}{lrrr}\mathrm{H} & -1.451898 & -0.913425 & -3.145165 \\ \mathrm{C} & -0.407218 & -1.068412 & -2.896079 \\ \mathrm{C} & -0.058681 & -1.265434 & -1.657180 \\ \mathrm{H} & 0.344870 & -1.089277 & -3.678050 \\ \mathrm{~N} & 0.286726 & -1.372045 & -0.459090 \\ \mathrm{H} & 0.313633 & -2.317486 & -0.069396 \\ \mathrm{H} & 0.609997 & -0.355029 & 0.246606 \\ \mathrm{~N} & 0.911085 & 0.674934 & 0.943315 \\ \mathrm{H} & 1.862350 & 1.051106 & 0.949035 \\ \mathrm{C} & 0.069128 & 1.259357 & 1.661402 \\ \mathrm{C} & -0.865891 & 1.809345 & 2.381417 \\ \mathrm{H} & -1.021320 & 1.482995 & 3.404591 \\ \mathrm{H} & -1.466328 & 2.611759 & 1.965453\end{array}$

\section{8. $\mathrm{CH}_{2} \mathrm{CH}=\mathrm{NH}^{+}(\mathrm{MP} 2)$}

$\mathrm{H} \quad 0.395111-0.007268 \quad-2.111936$

$\begin{array}{llll}\text { C } & -0.161660 & -0.006520 & -1.174178\end{array}$

$\begin{array}{llll}\text { C } & 0.478712 & 0.002609 & 0.097638\end{array}$

$\mathrm{H} \quad \begin{array}{llll}\mathrm{C} & -1.252681 & -0.012236 & -1.192249\end{array}$

$\begin{array}{llll}\mathrm{H} & 1.564436 & 0.008434 & 0.191697\end{array}$

$\begin{array}{llll}\mathrm{N} & -0.384661 & 0.003411 & 1.081633\end{array}$

$\mathrm{H} \quad 0.083453 \quad 0.010654 \quad 2.000297$

38. $\mathrm{CH}_{2} \mathrm{CH}=\mathrm{NH}^{+}$(DFT)

H $0.4016859089-0.0076730245-2.1077070659$

C $-0.1644895647-0.0061990354-1.1748593488$

C 0.46988545430 .0027584990 .0965789304

H $-1.2553977186-0.0122765229-1.208621222$

H $1.5579097584 \quad 0.00852717910 .1866400334$

$\begin{array}{lllll}\mathrm{N} & -0.3762871721 & 0.0034663687 & 1.0899211224\end{array}$

H 0.08940333370 .0104805362 .0109495505 
39. $\mathrm{CH}_{2} \mathrm{CHNH}^{+}$(nitrogen bridged) (MP2)

$\begin{array}{lllc}\mathrm{C} & 0.671633 & 0.000000 & -0.437818 \\ \mathrm{C} & 0.012811 & 0.000000 & 0.895271 \\ \mathrm{H} & -0.070710 & -0.935525 & 1.437448 \\ \mathrm{~N} & -0.584523 & 0.000000 & -0.489878 \\ \mathrm{H} & -0.070710 & 0.935525 & 1.437448 \\ \mathrm{H} & -1.408635 & 0.000000 & -1.095838 \\ \mathrm{H} & 1.535057 & 0.000000 & -1.094632\end{array}$

39. $\mathrm{CH}_{2} \mathrm{CHNH}^{+}$(nitrogen bridged) (DFT)

C 0.6676626976 0. -0.4396060452

C $0.0089456313 \quad 0.0 .8938193401$

H $-0.0688015622-0.93466419641 .4426065494$

$\mathrm{N}-0.5831972428$ 0. -0.4910149923

H -0.06880156220 .93466419641 .4426065494$

H -1.4043399919 0. -1.0993974892

H 1.5334550304 0. -1.0970129122

40. $\mathrm{CH}_{3} \mathrm{~N} \equiv \mathrm{CH}^{+}(\mathrm{MP} 2)$

H $0.5202648975 \quad 0.9011252358 \quad 1.5600985979$

C 0. 0. 1.235569614

H $0.5202648975 \quad-0.90112523581 .5600985979$

$\mathrm{N}$ 0. $0 .-0.2102262802$

H -1.0405297949 0. 1.5600985979

C 0. 0. -1.3635199139

H $0.0 .-2.4410100325$

40. $\mathrm{CH}_{3} \mathrm{~N} \equiv \mathrm{CH}^{+}$(DFT)

H 0.51978980880 .90030235811 .5599757504

C 0. 0. 1.2233320264

H $0.5197898088 \quad-0.90030235811 .5599757504$

$\mathrm{N}$ 0. $0 .-0.2123254072$

H $-1.0395796176 \quad 0.1 .5599757504$

C 0. 0. -1.3551715832

H $0.0 . \quad-2.4356629319$

41. $\mathrm{HC} \equiv \mathrm{C}-\mathrm{NH}_{3}{ }^{+}(\mathrm{MP} 2)$

H 0. 0. -2.5252908898

C 0. 0. -1.455529724

C 0. 0. -0.2468758084

N 0. 0.1 .1621785688

H $0.48038569910 .832052438 \quad 1.5348247009$

H $0.4803856991 \quad-0.8320524381 .5348247009$

H $-0.9607713981 \quad 0.1 .5348247009$ 

41. $\mathrm{HC} \equiv \mathrm{C}-\mathrm{NH}_{3}{ }^{+}$(DFT)
H 0. $0 .-2.5234283344$
C 0. 0. -1.451436548
C 0. 0. -0.2515066274
N 0. 0. 1.1577109742
H $0.48071907910 .8326298692 \quad 1.535651154$
H $0.4807190791 \quad-0.83262986921 .535651154$
H $-0.9614381583 \quad 0.1 .535651154$

42. diazomethane (MP2)

$\begin{array}{lrrr}\mathrm{H} & 0.961151 & 0.000000 & -1.642621 \\ \mathrm{C} & 0.000000 & 0.000000 & -1.156342 \\ \mathrm{~N} & 0.000000 & 0.000000 & 0.159334 \\ \mathrm{H} & -0.961151 & 0.000000 & -1.642621 \\ \mathrm{~N} & 0.000000 & 0.000000 & 1.301137\end{array}$

42. diazomethane (DFT)

H $0.95373616050 .-1.6476763847$

C $0.0 .-1.1407927933$

N 0. 0.0 .1548329036

H -0.9537361605 0. -1.6476763847

N 0. 0. 1.3001996592

43. 1-diazirene (MP2)

C $0.0001536697-0.00000025790 .8083895169$

H $-0.0012227834-0.9373826643 \quad 1.3462388175$

H $0.00106110890 .9373829044 \quad 1.3462384986$

N $0.6235823052-0.0000433588$-0.5388806962

N $-0.62369092570 .0000435456-0.5386642206$

43. 1-diazirine (DFT)

$\begin{array}{llll}\text { C } & -0.000158637 & 0.0000000441 & 0.8050667778\end{array}$

H $-0.0003727524 \quad-0.93529943131 .3528881496$

H $0.0005412010 .9352994623 \quad 1.3528878407$

N $0.6131907124-0.0010700243-0.543704275$

N $-0.61331714940 .0010702968-0.5438165769$

44. 2-diazirene (MP2)

N $-0.255013297 \quad 0.0312420793-0.8862711186$

$\begin{array}{llll}\text { C } & -0.0896204456 & 0.5031360031 & 0.4364385514\end{array}$

N $0.281199381-0.69236298570 .5651127787$

H $-0.2697363961 \quad 1.3992527158 \quad 1.0148498818$

H $0.6241564820 .2097776099-1.3853728108$ 

44. 2-diazirine (DFT)
$\mathrm{N}-0.2448276983 \quad 0.0180058119 \quad-0.8772489493$
$\begin{array}{lllll}\text { C } & -0.0924514525 & 0.4988261315 & 0.4400631474\end{array}$
N $0.2721112697 \quad-0.68424422140 .5582354939$
H $-0.26623982891 .401697001 \quad 1.0162756877$
H $0.6223934344 \quad 0.2167606994-1.3925680972$

45. carbodiimide (MP2)

$\mathrm{N}-0.0589386427 \quad-0.0629253143 \quad-1.2272522303$

C 0.02104597110 .02256904130 .

$\mathrm{N}-0.0586606775 \quad-0.0631845192 \quad 1.2272522304$

H $0.8067431829-0.0536789553-1.7550234213$

H -0.10982376790 .8010335421 .7550234207$

45. carbodiimide (DFT)

$\mathrm{N}-0.0484755455 \quad-0.0618789414-1.2235640794$

C 0.01909026630 .02047180460 .

N - $0.058346722 \quad-0.0526739182 \quad 1.2235640795$

H $0.8036602882-0.0596469978-1.7736821405$

H $-0.11556222320 .7975418473 \quad 1.7736821398$

$\begin{array}{lrrr}\text { 46. } \mathrm{CH}_{3} \mathrm{~N} \equiv \mathrm{N}^{+}(\mathrm{MP} 2) & & \\ \mathrm{H} & 0.524890 & 0.909136 & 1.491254 \\ \mathrm{C} & 0.000000 & 0.000000 & 1.193020 \\ \mathrm{H} & 0.524890 & -0.909136 & 1.491254 \\ \mathrm{~N} & 0.000000 & 0.000000 & -0.271089 \\ \mathrm{H} & -1.049780 & 0.000000 & 1.491254 \\ \mathrm{~N} & 0.000000 & 0.000000 & -1.390609\end{array}$

46. $\mathrm{CH}_{3} \mathrm{~N} \equiv \mathrm{N}^{+}$(DFT)

H 0.52469306540 .90879504761 .49112297

C 0. 0. 1.1801937876

H $0.5246930654-0.90879504761 .49112297$

$\mathrm{N} 0.0 .-0.2723629387$

$\mathrm{H}-1.0493861308 \quad 0.1 .49112297$

N 0. $0 .-1.3762693837$

47. diazomethane $+\mathrm{CH}_{3} \mathrm{~N} \equiv \mathrm{N}^{+}$, ion-molecule complex (MP2)

$\mathrm{H} \quad 1.334496 \quad 1.634417 \quad 0.894116$

C $\quad \begin{array}{llll}\text { C } & 1.230768 & 1.030462 & -0.009299\end{array}$

$\begin{array}{lllll}\mathrm{H} & 0.315548 & 0.376884 & -0.004864\end{array}$

$\mathrm{N} \quad \begin{array}{llll}2.342175 & 0.102117 & -0.000603\end{array}$

$\mathrm{H} \quad \begin{array}{llll}\mathrm{H} & 1.336258 & 1.619499 & -0.922313\end{array}$

$\mathrm{N} \quad 3.154861 \quad-0.670090 \quad 0.006557$

C $\quad-1.258184-0.962920 \quad 0.004723$

$\mathrm{N} \quad-2.354748 \quad-0.168012 \quad 0.001789$ 


\begin{tabular}{|c|c|c|}
\hline $\mathrm{H}$ & -1.231830 & -1.538980 \\
\hline $\mathrm{H}$ & -1.233911 & -1.548663 \\
\hline $\mathrm{N}$ & -3.193155 & 0.600498 \\
\hline
\end{tabular}

48. diazomethane $+\mathrm{CH}_{3} \mathrm{~N} \equiv \mathrm{N}^{+}$, proton transfer ts (MP2)

$\begin{array}{llll}\mathrm{H} & -1.160021 & 1.663951 & 0.252577\end{array}$

$\begin{array}{llll}\text { C } & -1.250049 & 0.814847 & -0.427745\end{array}$

$\begin{array}{llll}\mathrm{H} & -0.529004 & -0.284078 & 0.063462\end{array}$

$\mathrm{N} \quad-0.700649 \quad 1.175075 \quad-1.643341$

$\mathrm{H} \quad-2.283051 \quad 0.505475 \quad-0.596953$

$\mathrm{N} \quad \quad-0.143233 \quad 1.347270 \quad-2.615358$

$\begin{array}{llll}\text { C } & 0.081429 & -1.442282 & 0.567870\end{array}$

$\begin{array}{llll}\mathrm{N} & 0.896269 & -1.070046 & 1.619833\end{array}$

H $\quad 0.704339-1.908518 \quad-0.197984$

$\mathrm{H} \quad-0.695343 \quad-2.104764 \quad 0.954111$

$\mathrm{N} \quad \begin{array}{llll}1.515443 & -0.610506 & 2.450871\end{array}$

\section{9. $\mathrm{CH}_{2} \mathrm{NNH}^{+}(\mathrm{MP} 2)$}

$\mathrm{H} \quad 0.962217 \quad-0.078126 \quad-1.716544$

$\begin{array}{llll}\mathrm{C} & -0.001252 & -0.057831 & -1.209163\end{array}$

$\begin{array}{llll}\mathrm{N} & 0.000138 & 0.031473 & 0.047074\end{array}$

$\mathrm{H} \quad-0.965819 \quad-0.075845 \quad-1.714544$

$\begin{array}{llll}\mathrm{N} & 0.001042 & -0.080256 & 1.234620\end{array}$

$\mathrm{H} \quad 0.002854 \quad 0.842437 \quad 1.714203$

\section{9. $\mathrm{CH}_{2} \mathrm{NNH}^{+}$(DFT)}

H $0.9564999179-0.0817379552-1.726414592$

C $-0.0012599505-0.05873777-1.203149886$

N $0.0000137395 \quad 0.03466224720 .0461876138$

H $-0.9600641559-0.0797550893-1.7245836166$

N $0.0008041867 \quad-0.0709843576 \quad 1.2291747975$

H $0.0031862623 \quad 0.838404925 \quad 1.7344316834$

50. $\mathrm{CH}_{2} \mathrm{NNH}^{+}$, ts for inversion at $\mathrm{N} 1$ (MP2)

$\mathrm{H} \quad 0.968865 \quad 0.000000 \quad-1.720738$

C $\quad 0.000000 \quad 0.000000 \quad-1.229829$

$\begin{array}{llll}\mathrm{N} & 0.000000 & 0.000000 & 0.044736\end{array}$

$\mathrm{H} \quad-0.968865 \quad 0.000000 \quad-1.720738$

$\begin{array}{llll}\mathrm{N} & 0.000000 & 0.000000 & 1.186116\end{array}$

$\begin{array}{llll}\mathrm{H} & 0.000000 & 0.000000 & 2.204493\end{array}$

51. diazomethane $+\mathrm{CH}_{2} \mathrm{NNH}^{+}$, ion-molecule complex (MP2)

$\begin{array}{lrrr}\mathrm{C} & 1.511372 & 0.253054 & 2.872001 \\ \mathrm{H} & 2.324030 & -0.452208 & 3.020328 \\ \mathrm{~N} & -0.574025 & 0.087405 & -2.085776 \\ \mathrm{H} & 1.450216 & 1.235438 & 3.331342\end{array}$




$\begin{array}{lrrr}\mathrm{N} & 0.040623 & 0.228224 & -1.121514 \\ \mathrm{H} & -0.262233 & -0.206520 & 0.383793 \\ \mathrm{~N} & -0.352492 & -0.451528 & 1.458597 \\ \mathrm{~N} & 0.574565 & -0.087660 & 2.088840 \\ \mathrm{C} & -1.240033 & -0.054789 & -3.186887 \\ \mathrm{H} & -1.043255 & -0.944085 & -3.769061 \\ \mathrm{H} & -1.917488 & 0.742700 & -3.458109\end{array}$

52. diazomethane $+\mathrm{CH}_{2} \mathrm{NNH}^{+}$, proton transfer ts (MP2)

$\begin{array}{lrrr}\mathrm{C} & 1.363139 & 0.162425 & 2.972101 \\ \mathrm{H} & 2.157825 & -0.547809 & 3.166812 \\ \mathrm{~N} & -0.546918 & 0.114510 & -2.022532 \\ \mathrm{H} & 1.194423 & 1.082033 & 3.519573 \\ \mathrm{~N} & 0.229571 & 0.396215 & -1.204207 \\ \mathrm{H} & 0.000121 & 0.000031 & -0.000157 \\ \mathrm{~N} & -0.229431 & -0.396287 & 1.204108 \\ \mathrm{~N} & 0.546915 & -0.114524 & 2.022533 \\ \mathrm{C} & -1.363268 & -0.162365 & -2.971995 \\ \mathrm{H} & -1.194703 & -1.081997 & -3.519481 \\ \mathrm{H} & -2.157845 & 0.547998 & -3.166697\end{array}$

$\begin{array}{lccc}\text { 53. } \mathrm{CH}_{2}=\mathrm{NHN}^{+}(\mathrm{MP} 2) & & \\ \mathrm{H} & 1.287889 & 0.000000 & 1.258506 \\ \mathrm{C} & 0.212892 & 0.000000 & 1.129063 \\ \mathrm{H} & -0.506259 & 0.000000 & 1.935968 \\ \mathrm{~N} & -0.264867 & 0.000000 & -0.085464 \\ \mathrm{H} & -1.312000 & 0.000000 & -0.230429 \\ \mathrm{~N} & 0.158155 & 0.000000 & -1.305739\end{array}$

53. $\mathrm{CH}_{2}=\mathrm{NHN}^{+}$(DFT)

H $1.2862334902 \quad 0.1 .2756024471$

C 0.210995725 0. 1.1301205743

H $-0.5053287636 \quad 0.1 .9412595206$

$\mathrm{N}-0.248083514 \quad 0 .-0.0855634335$

$\mathrm{H}-1.3010048346 \quad 0 .-0.2378035265$

N $0.132997897 \quad 0 .-1.3217105821$

53a. $\mathrm{CH}_{2} \mathrm{NHN}^{+}$(nitrogen bridged) (MP2)

$\mathrm{N} 0.4485364380 .0 .5519415292$

C 0.12407545790 .0 .8584506423

H $0.2258243309 \quad-0.9500374676 \quad 1.3676250209$

$\mathrm{N}-0.7706968143 \quad 0 . \quad-0.3762720091$

H 0.22582433090 .95003746761 .3676250209

H $1.0590212252 \quad 0 .-1.3884591272$

53a. $\mathrm{CH}_{2} \mathrm{NHN}^{+}$(nitrogen bridged) (DFT) 
N $0.44535991620 .-0.5551760617$

C 0.13220147960 .0 .8568623041

H $0.2220572154 \quad-0.94927822251 .3747934384$

N $-0.763720725 \quad 0 .-0.3820651347$

H 0.22205721540 .94927822251 .3747934384

H 1.054629867 0. -1.3921799657

54. $\mathrm{H}_{2} \mathrm{~N}=\mathrm{C}=\mathrm{NH}^{+}$(MP2)

$\begin{array}{llll}\text { H } & 1.5360312214 & -0.8774049323 & 0.0231736643\end{array}$

N $1.02641536910 .0000072237 \quad-0.0100886149$

C $-0.26517874290 .0000118717-0.0207654628$

H 1.53604230550 .87741392740 .0231713212

$\mathrm{N}-1.4239003093-0.0000101335-0.1204302867$

H $-2.4094098448-0.00001795690 .1049393799$

54. $\mathrm{H}_{2} \mathrm{~N}=\mathrm{C}=\mathrm{NH}^{+}$(DFT)

H $1.5286709505 \quad-0.87628405320 .0342724156$

N 1.00931191390 .00000875350 .0058693637

C $-0.2742542485 \quad 0.0000232072-0.042634621$

H $1.5286937157 \quad 0.87628924720 .0342724576$

$\mathrm{N}-1.4289597146 \quad-0.0000162943 \quad-0.2116908768$

H - $2.3634626181-0.00002086030 .1799112619$

55. carbodiimide $+\mathrm{H}_{2} \mathrm{~N}=\mathrm{C}=\mathrm{NH}^{+}$, ion-molecule complex (MP2)

C $2.103687584-0.10154635150 .0021888929$

H $-3.4892663761 .047049018 \quad 1.1045463553$

N $2.8735651023 \quad 0.7442042103 \quad-0.2955613832$

H $1.4490173186-1.61162929561 .0912263291$

H $0.1559841356-0.8065090795 \quad 0.0513865128$

$\mathrm{N}-1.2725118202-0.6797682928-0.4861670575$

C $-2.12386371940 .1207848285-0.0237674901$

N $-2.7907058839 \quad 1.04334658140 .3717865764$

H $3.58320779090 .9617459017-0.9826765956$

H - $1.6004873644-1.2593917237-1.2568457709$

N $1.19288136-0.8858818822 \quad 0.4273468289$

56. carbodiimide $+\mathrm{H}_{2} \mathrm{~N}=\mathrm{C}=\mathrm{NH}^{+}$, proton transfer ts (MP2)

C $2.04097606560 .3527154106-0.2332635543$

H $-3.1832058111-2.02808159270 .4411471594$

N $2.90634139090 .1898848237 \quad 0.5726392301$

H $1.2293401723 \quad 0.2570668779-2.0004242924$

H $-0.15205233860 .4833055147-0.5803873256$

$\begin{array}{lllll}\mathrm{N} & -1.3430250169 & 0.6182433701 & -0.1576553399\end{array}$

$\begin{array}{llll}\text { C } & -2.0386828966 & -0.3600002983 & 0.2420141619\end{array}$

N - $-2.5566487885-1.30129272250 .7621426683$

H $3.56864614340 .8032067267 \quad 1.0298278822$ 
H $-1.7921565882 \quad 1.5315716721-0.147776493$

N $1.03842804420 .3498274039-1.0049680694$

57. $\mathrm{HNCHNH}^{+}$, (open, "sickle-shaped" form) (MP2)

H $1.0384364061 .0084531044-0.2237760771$

N 0.34898516430 .41838177640 .2576510251

$\begin{array}{llll}\text { C } & 0.1788715187 & -0.8605522324 & 0.0287767963\end{array}$

H $0.5520116189-1.835496795 \quad 0.3358377444$

N $-0.6926726974-0.3056622928-0.7773830919$

H $-1.6754409834-0.1294235597-0.5358216978$

58. $\mathrm{HNCHNH}^{+}$, (carbon-bridged form) (MP2)

H 0. 0. -1.7893728336

C 0. $0 .-0.7013416277$

N 0.79420181380 .20010685440 .3212613559

N $-0.7942018138-0.20010685440 .3212613559$

$\begin{array}{lllll}\mathrm{H} & -1.3840904435 & 0.5232313386 & 0.7498818084\end{array}$

H $1.3840904435 \quad-0.52323133860 .7498818084$

58. $\mathrm{HNCHNH}^{+}$, (carbon-bridged form) (DFT)

H 0. 0. -1.7975611677

C 0. 0. -0.7066110305

N $0.77444014550 .1942910447 \quad 0.3327824113$

N $-0.7744401455-0.19429104470 .3327824113$

H $-1.3843852728 \quad 0.5230147 \quad 0.7450896214$

H $1.3843852728-0.5230147 \quad 0.7450896214$

59. carbodiimide $+\mathrm{HNCHNH}^{+}$(open, "sickle-shaped" form), open N-N dimmer (MP2)

C $0.669182249-0.93585759330 .99738254$

N $0.38310953290 .2962487406 \quad 0.6966675348$

H $0.09538073530 .9534720602 \quad 1.4230162316$

N $0.53413299620 .6623519747-0.6442554151$

H $1.32349723751 .2920215577-0.7651771584$

C $-0.66850984161 .0440730812-1.3216758256$

H $-0.42787407191 .5340335006-2.2652522397$

N $0.9261868297-2.0401868475 \quad 1.2426793721$

$\mathrm{N}-1.87024919510 .8279754484-0.9835014758$

H $-1.95696703990 .3483304727-0.0842087651$

H 1.1496675498 -3.0018757317 1.4562515331

60. structure 59, (closed form), internal proton transfer ts (MP2)

H 0.0 .0 .8455389521

C $-0.0538430774 \quad 1.1958042127 \quad 0.2345734109$

H $-0.11157413383 .1952259357 \quad 0.9258367198$ 


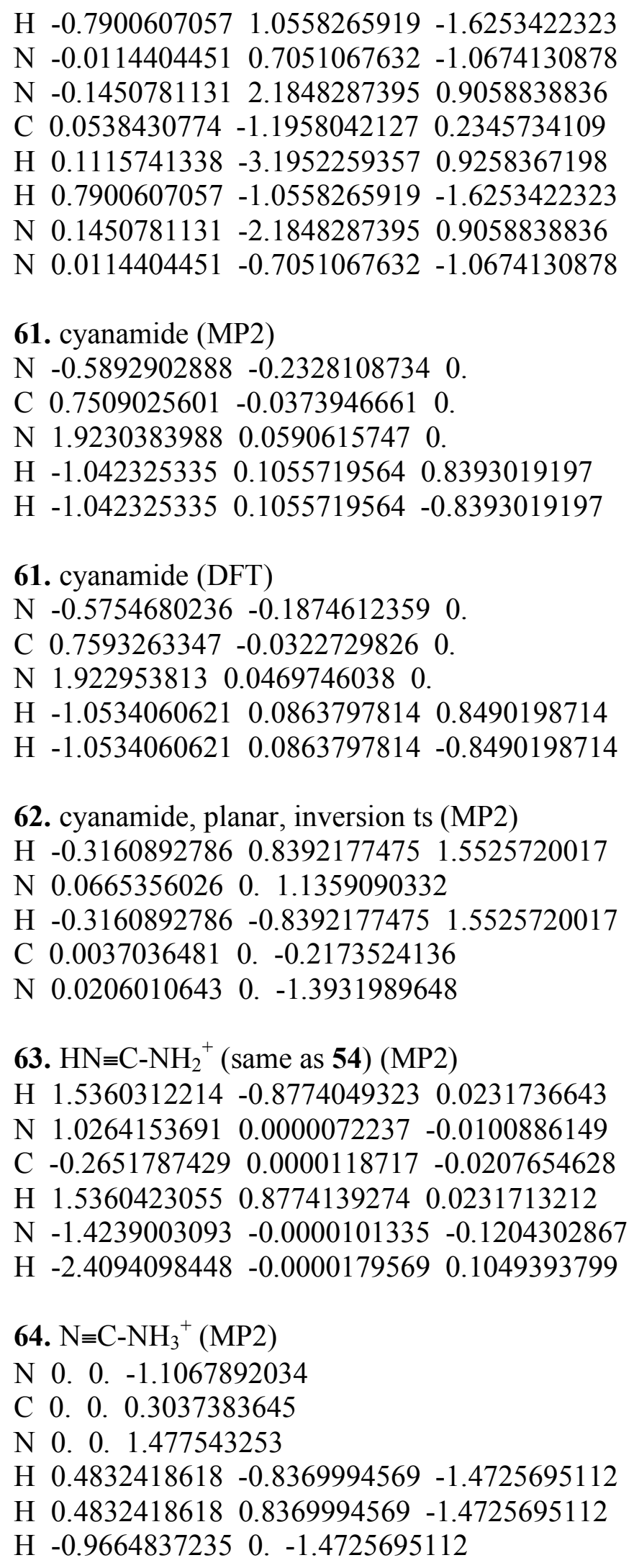




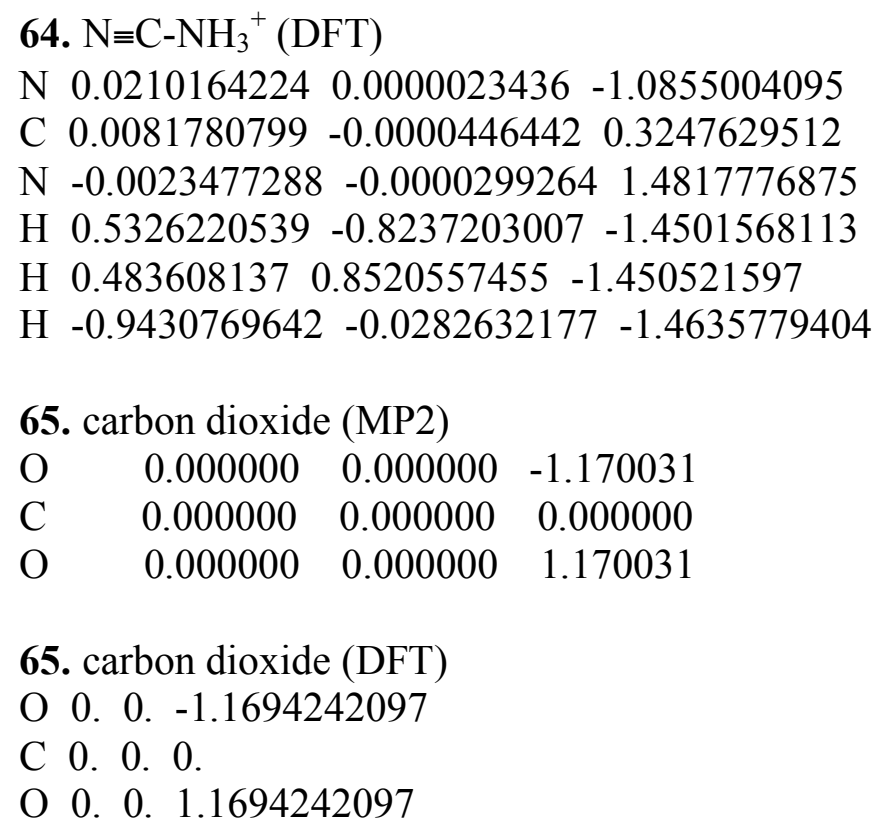

65. carbon dioxide (DFT)

O $0.0 . \quad-1.1694242097$

C 0. 0. 0 .

O 0.0 .1 .1694242097

66. carbon dioxide, cyclic (MP2)

$\begin{array}{lllll}\text { O } & 0.7924652117 & 0 . & 0.2888436337\end{array}$

C 0. 0. -0.77024969

O $\quad-0.7924652117$ 0. 0.2888436337

66. carbon dioxide, cyclic (DFT)

O $0.7742013746 \quad 0.0 .2959671737$

C 0. 0. -0.78449677

O -0.7742013746 0. 0.2959671737

67. $\mathrm{OCOH}^{+}(\mathrm{MP} 2)$

O $0.10134186910 .-1.1164556128$

C -0.01390541310 .0 .1067072299$

O 0.0001978864 0. 1.2423438003

H -0.7288855657 0. -1.6473488798

67. $\mathrm{OCOH}^{+}(\mathrm{DFT})$

O $0.0813653473 \quad 0 .-1.1021632026$

C -0.01624640640 .0 .119151644$

O 0.0147413334 0. 1.2512054429

H $-0.7211114976 \quad 0 .-1.6829473467$

68. carbon dioxide $+\mathrm{OCOH}^{+}$, ion-molecule complex (MP2)

C $-1.3775360692-0.8552901317 \quad 1.4228222954$

$\begin{array}{lllll}\text { O } & -1.7395824122 & -1.6001420861 & 2.2131821134\end{array}$

O $-1.0594733555-0.03960935430 .5993224171$

H $\quad-0.0543491666 \quad 0.01974972070 .0136237185$

$\begin{array}{lllll}\text { O } & 0.9975208702 & 0.0769891141 & -0.5950167886\end{array}$ 
$\begin{array}{llll}\text { C } & 1.3839292207 & 0.8518774665 & -1.4232966748\end{array}$

$\begin{array}{lllll}\text { O } & 1.8035336797 & 1.5628531101 & -2.2188349221\end{array}$

69. carbon dioxide $+\mathrm{OCOH}^{+}$, proton transfer ts (MP2)

C $-1.3883316984-0.8687834901 \quad 1.406006235$

O $-1.7571352874-1.55158321092 .248826562$

O $-1.0630728637-0.12892048680 .5189509322$

$\mathrm{H}-0.0006802342-0.0000345405-0.0000801176$

$\begin{array}{llll}\text { O } & 1.0622046499 & 0.1287526721 & -0.5194644292\end{array}$

$\begin{array}{llll}\text { C } & 1.3885154471 & 0.8690676604 & -1.4057001177\end{array}$

$\begin{array}{llll}\text { O } & 1.7579507189 & 1.5515422154 & -2.2485326383\end{array}$

70. $\mathrm{OCOH}^{+} \rightarrow{ }^{+} \mathrm{HOCO}$, internal proton transfer ts (MP2)

H 0. 0. -1.1459710062

C 0. 0.0 .0866932512

O $1.2010204178 \quad 0.0 .0391132187$

O -1.2010204178 0. 0.0391132187

71. $\mathrm{OCHO}^{+}$, (center-protonated $\mathrm{CO}_{2}$ ) (MP2)

H 0. 0. -1.6866187159

C 0. 0. -0.5956833968

$\begin{array}{lllll}\text { O } & -0.866514597 & 0 . & 0.3287949436\end{array}$

$\begin{array}{lllll}\text { O } & 0.866514597 & 0 . & 0.3287949436\end{array}$

71. $\mathrm{OCHO}^{+}$, (center-protonated $\left.\mathrm{CO}_{2}\right)(\mathrm{DFT})$

H 0. 0. -1.7105718486

C 0. 0. -0.6142972716

O $-0.8238140771 \quad 0.0 .3500784473$

O $0.8238140771 \quad 0.0 .3500784473$

72. carbon dioxide $+\mathrm{OCHO}^{+}$, open $\mathrm{O}-\mathrm{O}$ dimer (MP2)

C $-1.454543609-0.224247453 \quad 0.8407656266$

O $-0.9319370719-0.1262961969-0.2783014172$

O $0.5521926372-0.1675912406-0.0225427206$

C $1.2807734422 \quad 0.4019065398 \quad-1.1805184101$

O $-2.0078089705-0.3162934594 \quad 1.8302345926$

$\begin{array}{llll}\text { O } & 2.4417234308 & 0.3854308397 & -1.0271969792\end{array}$

H $0.6092607950 .7320459368-1.9790311038$

73. carbon dioxide $+\mathrm{OCHO}^{+}$, (closed form), internal proton transfer ts (HF); cx (MP2)

O -0.7667272753 0. -2.2091369795

O $1.01536176860 .-0.7151074597$

O 1.01300157390 .0 .7184469181

C -0.2521495595 0. 1.1705813615

C -0.2482940444 0. -1.171405108

O -0.7739974752 0. 2.2066002636 
H -0.8984471132 0. -0.0014794605

74. isocyanic acid, $\mathrm{HN}=\mathrm{C}=\mathrm{O}$ (MP2)

H -0.5908252793 0. -1.8386266962

N $0.15972342550 .-1.1659519906$

C -0.03254404120 .0 .0439462546$

O -0.0414968065 0. 1.2170766379

74. isocyanic acid, $\mathrm{HN}=\mathrm{C}=\mathrm{O}$ (DFT)

H -0.5820142123 0. -1.8589472646

N $0.14137829690 .-1.1570284698$

C -0.02937992850 .0 .0488369596$

O -0.0351268576 0. 1.2235829805

75. $\mathrm{H}_{2} \mathrm{~N}-\mathrm{C} \equiv \mathrm{O}^{+}$(MP2)

N $0.6229028388-0.0691748673-0.9402667371$

C $-0.0819144407 \quad 0.009096842 \quad 0.1236497917$

$\begin{array}{llll}\text { O } & -0.7095791539 & 0.0788004567 & 1.0711035832\end{array}$

H $1.6413669266-0.0646312835-0.8785075726$

H $0.1664330771-0.1361293504-1.8503526832$

\section{5. $\mathrm{H}_{2} \mathrm{~N}-\mathrm{C} \equiv \mathrm{O}^{+}$(DFT)}

N $0.6199971763-0.0688521776-0.9358805422$

C -0.08256554940 .00916910290 .1246320049$

O $-0.70919021180 .0787572787 \quad 1.070516682$

H $1.6426324799-0.0648226025-0.8811080969$

Н $0.1683363482-0.1362898414-1.8525341739$

76. $\mathrm{HN} \equiv \mathrm{COH}^{+}(\mathrm{MP} 2)$

H $-1.8695485376-0.0932103817-1.2095173105$

N $-1.021333839-0.0562644406-0.6586510297$

C $-0.0518033613 \quad 0.012749592 \quad-0.0366248737$

$\begin{array}{llll}\text { O } & 0.9859515121 & -0.0495228755 & 0.6572595572\end{array}$

H 1.44209348160 .80674691760 .7817473025

76. $\mathrm{HN} \equiv \mathrm{COH}^{+}$(DFT)

H $-1.8743901803-0.103006837-1.2106957425$

N $-1.0266213665-0.0586452178 \quad-0.6616253546$

C $-0.06044032790 .0179006454-0.0433590551$

$\begin{array}{lllll}\text { O } & 0.9761174002 & -0.0356522311 & 0.6479594723\end{array}$

H 1.47069373040 .79990245220 .8019343258

77. $\mathrm{HNCHOH}^{+}(\mathrm{MP} 2)$

$\mathrm{H} 1.70095858610 .0 .1513195431$

C $0.61807594660 .-0.0569432716$ 

$\mathrm{N}-0.1995331604 \quad 0.0 .9175158121$
O -0.3545346935 0. -0.8952854579
$\mathrm{H}-1.1764045946$ 0. 1.2326521516
78. $\mathrm{H}_{2} \mathrm{C}-\mathrm{B}-\mathrm{CH}_{2}^{-}(\mathrm{MP} 2)$
C 0. 0. -1.4378068473
B 0. 0. 0 .
C 0. 0. 1.4378068473
H $-0.75135461140 .5261987913 \quad-2.0280005437$
H $0.7513546114-0.5261987913-2.0280005437$
H $-0.5260717941-0.75144353572 .0280005437$
H $0.5260717941 \quad 0.75144353572 .0280005437$
78. $\mathrm{H}_{2} \mathrm{C}-\mathrm{B}-\mathrm{CH}_{2}^{-}$(DFT)
C 0. 0. -1.4355226433
B 0. 0. 0 .
C 0. 0. 1.4355226433
H $-0.7486397167 \quad 0.5246814604-2.03333372$
H $0.7486397167-0.5246814604-2.03333372$
H $-0.5240392042 \quad-0.74908942912 .03333372$
H 0.52403920420 .74908942912 .03333372

79. $\mathrm{H}_{2} \mathrm{C}-\mathrm{B}-\mathrm{CH}_{2}{ }^{-}$, planar, rotational ts (MP2)

C -1.4525987225 0. 0.0000117241

B 0. 0. -0.0000009312

C 1.4525987225 0. 0.0000117241

H -2.0324861735 0. 0.921176322

H $-2.0323619756 \quad 0 .-0.9212443388$

H 2.03248617350 .0 .921176322

H $2.03236197560 .-0.9212443388$

80. $\mathrm{H}_{3} \mathrm{C}-\mathrm{B}-\mathrm{CH}_{2}$, end-protonated 79 (MP2)

H $0.6359101521 \quad 1.1043789925$ - 1.5494483783

C $0.6694353841 \quad 1.1600276571 \quad-0.4524159461$

H $1.72201103441 .1944179735-0.1616499532$

B $-0.0533191472 \quad-0.09354141490 .0645216241$

H $0.17265233662 .0880584982 \quad-0.1597779225$

C $-0.7161083324-1.2412459935 \quad 0.4891959318$

H $-0.8526610333-1.4737439682 \quad 1.5417891814$

H - $1.1312790644-1.9580944035-0.2142009623$

80. $\mathrm{H}_{3} \mathrm{C}-\mathrm{B}-\mathrm{CH}_{2}$, end-protonated 79 (DFT)

H $0.6408924798 \quad 1.1128391929$ - 1.5522321651

C $0.667069411 .1560123304-0.4527903887$

H $1.72240272041 .1965133952 \quad-0.1619324657$

B $-0.054206536 \quad-0.0939409948 \quad 0.0627613979$ 
H $0.17475484442 .0898529308-0.1602912088$

C $-0.7155943787-1.2385721588 \quad 0.4885127523$

H $-0.8547811396-1.47866805491 .5406006231$

H $-1.1338960704-1.9637792996-0.2066149702$

81. $\mathrm{H}_{3} \mathrm{C}-\mathrm{B}-\mathrm{CH}_{2}$, ts for ring closure to borirane, 82 (MP2)

H 0. 0. -1.8205244599

B $0.0 . \quad-0.626881479$

C $-1.2350989995 \quad 0.0 .2191028327$

C $1.2350989995 \quad 0.0 .2191028327$

H $1.1622899743 \quad 0.1 .3096557202$

H 2.2640388818 0. -0.1468067888

H -2.2640388818 0. -0.1468067888

H -1.1622899743 0. 1.3096557202

82. borirane (boracyclopropane) (MP2)

H 0. 0. -2.1528775986

B 0. $0 .-0.9716337313$

$\begin{array}{llll}\text { C } & 0.7769876471 & 0 . & 0.3545649033\end{array}$

C -0.77698764710 .0 .3545649033$

H 1.27491680690 .90734962630 .689066854

H $1.2749168069-0.90734962630 .689066854$

H $-1.2749168069-0.90734962630 .689066854$

H -1.27491680690 .90734962630 .689066854$

82. borirane (boracyclopropane) (DFT)

H 0. 0. -2.1525730027

B $0.0 . \quad-0.9688762238$

C $0.778045485 \quad 0.0 .3517033838$

C $-0.778045485 \quad 0.0 .3517033838$

H $1.28271677240 .9054147348 \quad 0.6897320866$

H $1.2827167724-0.9054147348 \quad 0.6897320866$

H $-1.2827167724 \quad-0.90541473480 .6897320866$

H $-1.28271677240 .9054147348 \quad 0.6897320866$

83. $\mathrm{H}_{2} \mathrm{C}-\mathrm{B}-\mathrm{CH}_{2}{ }^{-}+\mathrm{H}_{3} \mathrm{C}-\mathrm{B}-\mathrm{CH}_{2}$, proton transfer ts (MP2)

H $0.0002001642 \quad-0.0002017380 .0000009923$

C 0.0657353780 .07666990261 .4211788842

H $1.1264924216-0.15029894461 .546697587$

B $-0.9260055244 \quad-0.90210550261 .8851296012$

H $-0.1524882475 \quad 1.1404856827 \quad 1.5358502678$

C $-1.9166682131-1.88145462962 .1542883264$

H - $2.2120787734-2.16570517123 .1620295679$

H $-2.4364359888-2.40321051821 .3515952933$

C $-0.0652438792-0.077165574-1.4211741624$

B $0.92599477150 .9021164085-1.8851296371$ 
H $-1.12611712040 .1492365431-1.546733454$

H $0.1535471113-1.1408695942-1.5358020914$

C $1.9161539134 \quad 1.8819732524 \quad-2.1542930404$

H $2.21145422832 .1663346305-3.1620353098$

H $2.43561677422 .4040368718-1.3516027208$ 
Table S3. Selected Geometric Features of Compounds in This Study ${ }^{\mathrm{a}}$

Structure (point group) $)^{\mathrm{b}} \quad \mathrm{d}(\mathrm{X}-\mathrm{H})^{\mathrm{c}}, \AA \quad \Phi\left(\mathrm{X}-\mathrm{H}-\mathrm{X}^{\prime}\right)^{\mathrm{d}}$, deg. $\quad$ other features, $\AA$ or deg.

1 allene (D2)

2 propyne $(\mathrm{C} 3 \mathrm{v})$

3 cyclopropene $(\mathrm{C} 2 \mathrm{v})$

4 2-propenyl $\left.{ }^{+}(\mathrm{C} 1 / \mathrm{C} 2 \mathrm{v})\right)$

$51+4$ ts $(\mathrm{C} 1)$

6 allyl $^{+}(\mathrm{C} 2 \mathrm{v})$

7 allyl $^{+}$(rot. ts) $)^{\mathrm{e}}(\mathrm{Cl} / \mathrm{Cs})$

$84 \rightarrow 6(t s)^{\mathrm{f}}(\mathrm{C} 1 / \mathrm{Cs})$

$91+6(\mathrm{ts})^{\mathrm{g}}(\mathrm{C} 1)$

10 cyclopropyl $^{\text {th }}(\mathrm{Cs})$

11 ketene $(\mathrm{C} 2 \mathrm{v})$

12 ethynol (Cs)

13 2-oxirene (C2v)

14 acetyl $^{+}(\mathrm{C} 3 \mathrm{v}) \quad 1.096$

$1511+14 \mathrm{cx}^{\mathrm{i}}(\mathrm{C} 1)$

$1611+14$ ts $^{\mathrm{i}}(\mathrm{C} 1)$

$2.126,1.125$

$1.405,1.406$

$17 \mathrm{CH}_{2}=\mathrm{C}=\mathrm{OH}^{+}(\mathrm{C} 1 / \mathrm{Cs}) \quad 0.986$

1817 rotational ts $(\mathrm{Cs}) \quad 0.978$

$19 \mathbf{1 1}+\mathbf{1 7} \mathrm{cx}^{\mathrm{j}}(\mathrm{C} 1 / \mathrm{C} 2) \quad 1.200,1.199$

$2011+17 t^{\mathrm{j}}(\mathrm{C} 1 / \mathrm{C} 2)$

$1.200,1.199$

$21 \mathrm{CH}_{2} \mathrm{CH}=\mathrm{O}^{+}$O-bridged 1.089 $(\mathrm{Cl} / \mathrm{Cs})$

2221 rotational ts ${ }^{\mathrm{k}}(\mathrm{Cs}) \quad 1.095$

$2317 \rightarrow 21$ ts $(\mathrm{Cs})$

$1.386,1.285$
Allene and Isomers

159.4

55.5

151.7

Ketene and Isomers

$$
\begin{gathered}
\mathrm{d}(\mathrm{CC})=1.322, \\
\mathrm{~d}(\mathrm{CO})=1.168 \\
\mathrm{~d}(\mathrm{CC})=1.213, \\
\mathrm{~d}(\mathrm{CO})=1.316 \\
\mathrm{~d}(\mathrm{CC})=1.278, \\
\mathrm{~d}(\mathrm{CO})=1.503 \\
\mathrm{~d}(\mathrm{CC})=1.441, \\
\mathrm{~d}(\mathrm{CO})=1.127 \\
\text { dihedral }(\mathrm{CC} \mathrm{CC})=180 . \mathrm{O} \\
\text { dihedral(CC CC })=179.0 \\
\mathrm{~d}(\mathrm{CC})=1.283, \\
\mathrm{~d}(\mathrm{CO})=1.226 \\
\mathrm{~d}(\mathrm{CC})=1.278, \\
\mathrm{~d}(\mathrm{CO})=1.222 \\
\mathrm{~d}(\mathrm{CC})=1.295, \\
\mathrm{~d}(\mathrm{CO})=1.202 \\
\mathrm{~d}(\mathrm{CC})=1.295, \\
\mathrm{~d}(\mathrm{CO})=1.202 \\
\mathrm{~d}(\mathrm{CC})=1.434, \\
\mathrm{~d}(\mathrm{CO})=1.622, \\
\mathrm{~d}(\mathrm{C}=\mathrm{O})=1.243 \\
\mathrm{~d}(\mathrm{CC})=1.487, \\
\mathrm{~d}(\mathrm{C}=\mathrm{O})=1.227 \\
\mathrm{~d}(\mathrm{CC})=1.332, \\
\mathrm{~d}(\mathrm{CO})=1.221
\end{gathered}
$$

179.1

179.8

180.0

180.0 
27 ketenimine $(\mathrm{Cl} / \mathrm{Cs})$

\section{$\underline{\text { Ketenimine and Isomers }}$}

28 acetonitrile $(\mathrm{C} 3 \mathrm{v})$

29 methyl isonitrile $(\mathrm{C} 3 \mathrm{v})$

30 ethynamine $(\mathrm{C} 1 / \mathrm{Cs})$

31 2-azirine (Cs)

32 1-azirine (Cs)

$33 \mathrm{CH}_{3} \mathrm{C} \equiv \mathrm{NH}^{+}(\mathrm{C} 3 \mathrm{v}) 1.094(\mathrm{~N}-\mathrm{H}=1.014)$

$\mathbf{3 4} \mathbf{2 7}+\mathbf{3 3}$ ts $(\mathrm{C} 1 / \mathrm{C} 2) \quad 1.400,1.400$

180.0

$35 \mathrm{CH}_{2}=\mathrm{C}=\mathrm{NH}_{2}{ }^{+}(\mathrm{C} 2) \quad 1.022$

$3627+35 \mathrm{cx}^{1}(\mathrm{C} 1)$

$1.503,1.127$

178.4

$1.279,1.279 \quad 178.8$

$3727+35$ ts ${ }^{1}(\mathrm{C} 1 / \mathrm{C} 2)$

1.090

unbridged

$39 \mathrm{CH}_{2} \mathrm{CHNH}^{+}(\mathrm{Cs}) \quad 1.085$

N-bridged

$40 \mathrm{CH}_{3} \mathrm{~N} \equiv \mathrm{CH}^{+}(\mathrm{C} 3 \mathrm{v}) 1.077$

$41 \mathrm{HC} \equiv \mathrm{C}-\mathrm{NH}_{3}{ }^{+}(\mathrm{C} 3 \mathrm{v})$

$$
\begin{aligned}
& \mathrm{d}(\mathrm{CC})=1.318 \text {, } \\
& \mathrm{d}(\mathrm{CN})=1.235 \\
& \mathrm{~d}(\mathrm{CC})=1 / 463 \text {, } \\
& \mathrm{d}(\mathrm{CN})=1.174 \\
& \mathrm{~d}(\mathrm{CC})=1.426 \text {, } \\
& \mathrm{d}(\mathrm{NC})=1.184 \\
& \mathrm{~d}(\mathrm{CC})=1.217 \text {, } \\
& \mathrm{d}(\mathrm{CN})=1.363 \\
& \mathrm{~d}(\mathrm{CC})=1.287 \text {, } \\
& \mathrm{d}(\mathrm{CN})=1.524 \\
& \mathrm{~d}(\mathrm{CC})=1.452 \text {, } \\
& \mathrm{d}(\mathrm{CN})=1.564 \\
& \mathrm{~d}(\mathrm{C}=\mathrm{N})=1.270 \\
& \mathrm{~d}(\mathrm{CC})=1.449 \text {, } \\
& \mathrm{d}(\mathrm{CN})=1.154 \\
& \mathrm{~d}(\mathrm{CC})=1.379 \text {, } \\
& \mathrm{d}(\mathrm{CN})=1.183 \\
& \mathrm{~d}(\mathrm{CC})=1.292 \text {, } \\
& \mathrm{d}(\mathrm{CN})=1.261 \\
& \text { dihedral }(\mathrm{CN} \mathrm{NC})=93.1 \\
& \mathrm{~d}(\mathrm{CC})=1.424 \text {, } \\
& \mathrm{d}(\mathrm{CN})=1.309 \\
& \mathrm{~d}(\mathrm{CC})=1.487 \text {, } \\
& \mathrm{d}(\mathrm{CN})=1.508 \\
& \mathrm{~d}(\mathrm{C}=\mathrm{N})=1.257 \\
& \mathrm{~d}(\mathrm{CN})=1.446 \text {, } \\
& \mathrm{d}(\mathrm{N} \equiv \mathrm{C})=1.153 \\
& \mathrm{~d}(\mathrm{CC})=1.209 \text {, } \\
& \mathrm{d}(\mathrm{CN})=1.409
\end{aligned}
$$$$
\text { dihedral }(\mathrm{CN} \text { NC) }=93.9
$$

Diazomethane, Carbodiimide, and Isomers

42 diazomethane $(\mathrm{C} 2 \mathrm{v})$

43 1-diazirine (C2v)

44 2-diazirine $(\mathrm{C} 1)$

45 carbodiimide (C2)

$46 \mathrm{CH}_{3} \mathrm{~N} \equiv \mathrm{N}^{+}(\mathrm{C} 3 \mathrm{v}) \quad 1.092$

$4742+46 \mathrm{cx}^{\mathrm{m}}(\mathrm{Cl} / \mathrm{Cs})$

$4842+46$ ts $^{\mathrm{m}}(\mathrm{Cl} / \mathrm{C} 2)$
$2.067,1.125$

$1.403,1.403$
175.1

174.9

$$
\begin{aligned}
& d(\mathrm{CN})=1.316, \\
& d(\mathrm{NN})=1.142 \\
& d(\mathrm{CN})=1.485, \\
& \mathrm{~d}(\mathrm{NN})=1.247 \\
& \mathrm{~d}(\mathrm{CN})=1.414, \\
& \mathrm{~d}(\mathrm{NN})=1.708 \\
& \mathrm{~d}(\mathrm{C}=\mathrm{N})=1.258 \\
& \mathrm{~d}(\mathrm{CN})=1.233, \\
& \mathrm{~d}(\mathrm{NH})=1.014 \\
& d(\mathrm{CN})=1.464, \\
& d(\mathrm{NN})=1.110
\end{aligned}
$$$$
\text { dihedral }(\mathrm{NC} \mathrm{CN})=179.8
$$$$
\text { dihedral }(\mathrm{NC} \mathrm{CN})=106.6
$$ 


\begin{tabular}{|c|c|}
\hline $\begin{array}{l}49 \mathrm{CH}_{2} \mathrm{NNH}^{+}(\mathrm{C} 1 / \mathrm{Cs}) \\
\text { bent at } \mathrm{N} 1\end{array}$ & 1.040 \\
\hline $\begin{array}{l}50 \mathrm{CH}_{2} \mathrm{NNH}^{+}(\mathrm{C} 2 \mathrm{v}) \\
\text { linear at } \mathrm{N} 1 \text {, ts }\end{array}$ & 1.018 \\
\hline $5142+49 \mathrm{cx}^{\mathrm{n}}(\mathrm{C} 1 / \mathrm{Cs})$ & 96, \\
\hline $5242+49(\mathrm{ts})^{\mathrm{n}}(\mathrm{C} 1 / \mathrm{C} 2)$ & $88,1.2$ \\
\hline $53 \mathrm{CH}_{2}=\mathrm{NHN}^{+}(\mathrm{Cs})$ & 1.057 \\
\hline 53a $\mathrm{CH}_{2} \mathrm{NHN}^{+}$cyclic $(\mathrm{Cs})$ & 1.036 \\
\hline $54 \mathrm{NH}_{2}=\mathrm{C}=\mathrm{NH}^{+}(\mathrm{C} 1 / \mathrm{C} 2 \mathrm{v})$ & 1.015 \\
\hline $5545+54 \mathrm{cx}^{\mathrm{o}}(\mathrm{C} 1)$ & $1.532,1$ \\
\hline $5645+54$ ts $^{\circ}(\mathrm{C} 1 / \mathrm{C} 2)$ & $1.271,1.2$ \\
\hline $\begin{array}{l}57 \mathrm{HNCHNH}^{+}(\mathrm{Cl} / \mathrm{Cs}) \\
\text { open, "sickle" }\end{array}$ & 1.089 \\
\hline $\begin{array}{l}58 \mathrm{CHNHNH}^{+}(\mathrm{C} 2) \\
\text { C-bridged } \\
\mathbf{6 1} \text { cyanamide }(\mathrm{Cs})\end{array}$ & 1.088 \\
\hline 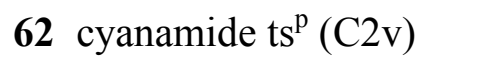 & \\
\hline $63 \mathrm{H}_{2} \mathrm{~N}-\mathrm{C} \equiv \mathrm{NH}^{+}(\mathrm{C} 2 \mathrm{v})$ & 1.010 \\
\hline $64 \mathrm{~N} \equiv \mathrm{C}-\mathrm{NH}_{3}{ }^{+}(\mathrm{C} 3 \mathrm{v})$ & 1.033 \\
\hline
\end{tabular}

$65 \mathrm{CO}_{2}\left(\mathrm{D}^{*} \mathrm{~h}\right)$

$66 \mathrm{CO}_{2}$ cyclic $(\mathrm{C} 2 \mathrm{v})$

$\begin{array}{llll}\mathbf{6 7} & \mathrm{OCOH}^{+}(\mathrm{Cs}) & 0.985 & \\ \mathbf{6 8} & \mathbf{6 5}+\mathbf{6 7} \mathrm{cx}(\mathrm{C} 1 / \mathrm{Cs}) & 1.217 .1 .165 & 179.7 \\ \mathbf{6 9} & \mathbf{6 5}+\mathbf{6 7} \text { ts }(\mathrm{C} 1 / \mathrm{C} 2 \mathrm{v}) & 1.190,1.190 & 179.9 \\ \mathbf{7 0} & \mathrm{OCOH}^{+} \mathrm{O}-\mathrm{H}-\mathrm{O} \text { ts }^{\mathrm{q}}(\mathrm{C} 2 \mathrm{v}) & 1.687,1.687 & 90.8 \\ & & & \\ \mathbf{7 1} & \mathrm{OCHO}^{+}(\mathrm{C} 2 \mathrm{v}) & 1.091 & \end{array}$

74 isocyanic acid (Cs)

$\underline{\text { Isocyanic Acid, } \mathrm{HN}=\mathrm{C}=\mathrm{O}}$
Carbon Dioxide

173.0

180.0

179.1

179.9

Carbon Dioxide

$\mathrm{d}(\mathrm{CN})=1.259$,
$\mathrm{d}(\mathrm{NN})=1.193$
$\mathrm{~d}(\mathrm{CN})=1.274$,
$\mathrm{d}(\mathrm{NN})=1.142$
dihedral $(\mathrm{NN} \mathrm{NN})=180.0$
dihedral $(\mathrm{NN} N \mathrm{NN})=180.0$
$\mathrm{~d}(\mathrm{CN})=1.306$,
$\mathrm{d}(\mathrm{NN})=1.292$
$\mathrm{~d}(\mathrm{CN})=1.447,1.525$
$\mathrm{~d}(\mathrm{CN})=1.293,1.159$
dihedral $(\mathrm{CN} \mathrm{NC})=84.8$
dihedral $(\mathrm{CN} \mathrm{NC})=86.4$
$\mathrm{~d}(\mathrm{CN})=1.341,1.333$
$\mathrm{~d}(\mathrm{CN})=1.310$,
$\mathrm{d}(\mathrm{NN})=1.638$
$\mathrm{~d}(\mathrm{NC})=1.355$,
$\mathrm{d}(\mathrm{C} \equiv \mathrm{N})=1.176$
$\mathrm{~d}(\mathrm{NC})=1.336$,
$\mathrm{d}(\mathrm{C} \equiv \mathrm{N})=1.177$
$\mathrm{~d}(\mathrm{NC})=1.293$,
$\mathrm{d}(\mathrm{C} \equiv \mathrm{N})=1.159$
$\mathrm{~d}(\mathrm{NC})=1.411$,
$\mathrm{d}(\mathrm{C} \equiv \mathrm{N})=1.174$

$\mathrm{d}(\mathrm{CO})=1.170$ $\mathrm{d}(\mathrm{CO})=1.323$, $\mathrm{d}(\mathrm{OO})=1.585$ $\mathrm{d}(\mathrm{CO})=1.229,1.136$

dihedral $($ CO OC $)=180.0$

dihedral $($ CO OC $)=179.9$ $\mathrm{d}(\mathrm{CO})=1.202$, $\mathrm{d}(\mathrm{CH})=1.233$ $\mathrm{d}(\mathrm{OO})=1.733$, $\Phi(\mathrm{OCO})=86$
$75 \mathrm{H}_{2} \mathrm{~N}-\mathrm{C} \equiv \mathrm{O}^{+}(\mathrm{Cs} / \mathrm{C} 2 \mathrm{v}) \quad 1.020$

$76 \mathrm{HN} \equiv \mathrm{COH}^{+}(\mathrm{C} 1)$

0.978

$77 \mathrm{HNCHO}^{+\mathrm{r}}$ (Cs)

$$
\begin{aligned}
& \mathrm{d}(\mathrm{NC})=1.225 \\
& \mathrm{~d}(\mathrm{CO})=1.173 \\
& \mathrm{~d}(\mathrm{NC})=1.279 \\
& \mathrm{~d}(\mathrm{CO})=1.139 \\
& \mathrm{~d}(\mathrm{NC})=1.154 \\
& \mathrm{~d}(\mathrm{CO})=1.250 \\
& \mathrm{~d}(\mathrm{NC})=1.272 \\
& \mathrm{~d}(\mathrm{CO})=1.284
\end{aligned}
$$


$78 \mathrm{CH}_{2} \mathrm{BCH}_{2}^{-}$(D2)

$79 \mathrm{CH}_{2} \mathrm{BCH}_{2}{ }^{-} \mathrm{ts}^{\mathrm{s}}(\mathrm{C} 2 \mathrm{v} / \mathrm{D} 2 \mathrm{~h})$

$80 \mathrm{CH}_{3} \mathrm{BCH}_{2}(\mathrm{C} 1 / \mathrm{C} 2 \mathrm{v}) \quad 1.099$

$81 \mathrm{CH}_{2} \mathrm{BHCH}_{2}$ ts $^{\mathrm{t}}(\mathrm{C} 2 \mathrm{v}) \quad 1.194$

82 borirane $^{\mathrm{t}}(\mathrm{C} 2 \mathrm{v}) \quad 1.187$

$\mathbf{8 3} \mathbf{7 8}+\mathbf{8 0}$ ts $^{\mathrm{u}}(\mathrm{C} 1 / \mathrm{C} 2 \mathrm{v}) \quad 1.425,1.425$
$\underline{\mathrm{CH}_{2}}-\mathrm{B}_{-}-\mathrm{CH}_{\underline{2}}{ }^{-}$

$\mathrm{d}(\mathrm{CB})=1.438$,
$\Phi(\mathrm{CBC})=180$
$\mathrm{~d}(\mathrm{CB})=1.453$,
$\Phi(\mathrm{CBC})=180$
$\Phi(\mathrm{CBC})=178.1$
$\mathrm{~d}(\mathrm{CB})=1.497$,
$\Phi(\mathrm{CBC})=111.2$
$\mathrm{~d}(\mathrm{CC})=1.554$,
$\mathrm{d}(\mathrm{CB})=1.537$
$180.0 \quad$ dihedral $(\mathrm{BC} \mathrm{CB})=180.0$

${ }^{a} \mathrm{MP} 2 / 6-311+\mathrm{G}^{* *}$ unless otherwise specified. ${ }^{\mathrm{b}} \mathrm{A}$ slash indicates the structure virtually has the higher symmetry. ${ }^{\mathrm{c}} \mathrm{d}(\mathrm{X}-\mathrm{H})$ is the bond distance between $\mathrm{H}$-donor atom $(\mathrm{X})$ and the hydrogen to be donated. It also denotes the partial bond distances between H-donor atoms (X) and acceptor atoms (X') and the donated hydrogen in complexes (cx) and transition states (ts). ${ }^{\mathrm{d}}$ Angle between $\mathrm{H}$-donor $(\mathrm{X})$, the donated hydrogen and $\mathrm{H}$-acceptor ( $\left.\mathrm{X}^{\prime}\right)$ in complexes and transition states. ${ }^{\mathrm{e}} \mathrm{MP} 2 / 6-311+\mathrm{G}^{* *} / \mathrm{HF} / 6-311+\mathrm{G}^{* *}$. The ts for rotation about a C-C bond in allyl cation. A "perpendicular" allyl cation could not be found at MP2/6-311+G**. ${ }^{\mathrm{f}}$ The transition state for the rearrangement of 2-propenyl ${ }^{+}$to allyl ${ }^{+}$and its reverse. An internal reaction coordinate (irc) calculation shows its connection to reactant and product. ${ }^{\mathrm{g}} \mathrm{A}$ hypothetical transition state model for proton transfer from $\mathrm{C} 2$ of allyl ${ }^{+}$to $\mathrm{C} 2$ of allene. A fully optimized structure was not obtained. Structure 9 has the partial C-H distances constrained to $1.420 \AA$. It is a second-order saddle point. ${ }^{\mathrm{h}}$ Cyclopropyl cation is a transition state for disrotatory ring opening to allyl ${ }^{+}$, shown by an irc calculation. The MP2/6-311+G** frequency calculation gives iv $=543 \mathrm{~cm}^{-1}$. 'Structure $\mathbf{1 5}$ is the C3-H-C3' hydrogen-bridged ion-molecule complex, and $\mathbf{1 6}$ is the corresponding proton-transfer ts. ${ }^{\mathrm{j}} \mathbf{1 9}$ is the $\mathrm{O}-\mathrm{H}-\mathrm{O}$ hydrogen-bridged ion-molecule complex, and $\mathbf{2 0}$ is the corresponding proton-transfer ts. ${ }^{\mathrm{k}} \mathrm{Structure} \mathbf{2 2}$ is flat. It is the ts for rotation about the $\mathrm{C}-\mathrm{C}$ bond of structure 21. At MP2 $/ 6-311+\mathrm{G}^{* *}$ iv $=206 \mathrm{~cm}^{-1}$.

${ }^{1}$ Structure $\mathbf{3 6}$ is the N-H-N hydrogen-bridged ion-molecule complex. Structure $\mathbf{3 7}$ is the corresponding proton-transfer ts. ${ }^{\mathrm{m}}$ Structure $\mathbf{4 7}$ is the $\mathrm{C}-\mathrm{H}-\mathrm{C}$ hydrogen-bridged ion-molecule complex, and $\mathbf{4 8}$ is the corresponding proton-transfer ts. "Structure $\mathbf{5 1}$ is the N1-H-N1' hydrogen-bridged ion-molecule complex, and $\mathbf{5 2}$ is the corresponding proton-transfer ts. ${ }^{\circ}$ Structure $\mathbf{5 5}$ is the N-H-N hydrogen-bridged ion-molecule complex, and $\mathbf{5 6}$ is the corresponding proton-transfer ts. ${ }^{\mathrm{p}}$ Structure $\mathbf{6 2}$ is the ts for flattening about the nitrogen. ${ }^{\mathrm{q}} \mathrm{Structure} \mathbf{7 0}$ is the ts for intramolecular proton transfer between the two oxygens. ${ }^{\mathrm{r}}$ Structure $\mathbf{7 7}$ has one imaginary frequency $=536 \mathrm{~cm}^{-1}$ at the HF level, but no imaginary frequency at the MP2 level. ${ }^{\mathrm{s}}$ Structure $\mathbf{7 9}$ is the ts for rotation about a $\mathrm{CB}$ bond in $\mathbf{7 8}$. ${ }^{\mathrm{t}}$ Transition state $\mathbf{8 1}$, protonated on boron, is a singlet with allyl ${ }^{+}$symmetry. Structure $\mathbf{8 2}$ has the shape of cyclopropyl cation. An irc calculation shows the two to be related by a disrotatory closure/opening. "Structure $\mathbf{8 3}$ is the ts for identity proton transfer between $\mathbf{7 8}$ and $\mathbf{8 0}$. 
Table S4. G3 Enthalpies ${ }^{\mathrm{a}}$

\begin{tabular}{|c|c|}
\hline 1 & -116.548314 \\
\hline 2 & -116.549305 \\
\hline 3 & -116.510992 \\
\hline 4 & -116.825425 \\
\hline 6 & -116.837838 \\
\hline 11 & -152.501751 \\
\hline 12 & -152.447077 \\
\hline 13 & -152.378882 \\
\hline 14 & -152.812361 \\
\hline 17 & -152.746039 \\
\hline 21 & -152.723038 \\
\hline 27 & -132.611294 \\
\hline 28 & -132.654081 \\
\hline 29 & -132.615177 \\
\hline 30 & -132.587066 \\
\hline 31 & -132.524612 \\
\hline 32 & -132.577251 \\
\hline 33 & -132.949846 \\
\hline 35 & -132.924892 \\
\hline 38 & -132.845025 \\
\hline 39 & -132.882034 \\
\hline 40 & -132.931604 \\
\hline 41 & -132.886989 \\
\hline 42 & -148.642145 \\
\hline 43 & -148.623353 \\
\hline 44 & -148.591862 \\
\hline 45 & -148.688423 \\
\hline 46 & -148.975276 \\
\hline 49 & -148.921922 \\
\hline 53 & -148.784347 \\
\hline $53 \mathbf{a}$ & -148.884971 \\
\hline 54 & -148.997164 \\
\hline 57 & -148.829694 \\
\hline 58 & -148.870401 \\
\hline 61 & -148.691947 \\
\hline 63 & -148.997086 \\
\hline 64 & -148.95463 \\
\hline
\end{tabular}




\begin{tabular}{ll}
$\mathbf{6 5}$ & -188.496481 \\
$\mathbf{6 6}$ & -188.273094 \\
$\mathbf{6 7}$ & -188.699486 \\
$\mathbf{7 1}$ & -188.542886 \\
$\mathbf{7 4}$ & -168.590636 \\
$\mathbf{7 5}$ & -168.863842 \\
$\mathbf{7 6}$ & -168.83839 \\
$\mathbf{7 7}$ & -168.715433 \\
$\mathbf{7 8}$ & -103.337936 \\
$\mathbf{8 0}$ & -103.907177 \\
$\mathbf{8 2}$ & -103.888894 \\
\hline
\end{tabular}

${ }^{a}$ Scaling factor of 0.9135 used for vibrations 